\title{
Efficacy and Safety of Metformin Versus the Other Oral Antidiabetic Drugs in Japanese Type 2 Diabetes Patients: A Network Meta-analysis
}

\author{
Rimei Nishimura $\cdot$ Mayumi Taniguchi · Tomomi Takeshima • \\ Kosuke Iwasaki
}

Received: September 13, 2021 / Accepted: October 21, 2021 / Published online: November 30, 2021

(c) The Author(s) 2021

\section{ABSTRACT}

Introduction: Although metformin is recommended as a first-line treatment for patients with type 2 diabetes (T2D) in Western countries, no specific treatment is recommended in Japan, and various agents are used. Metformin has long been used at low doses in Japan, and information regarding its effect at the maximum maintenance dose is lacking. Here, we compared the efficacy and safety of metformin at $1500 \mathrm{mg}$ /day with those of the other oral anti-diabetic drugs (OADs) approved in Japan.

Methods: Randomized controlled trials comparing a change in hemoglobin A1c (HbA1c) from baseline at 12 weeks or later $(\triangle \mathrm{HbA} 1 \mathrm{c})$ among OADs (including placebo) as a first-line

Supplementary Information The online version contains supplementary material available at https:// doi.org/10.1007/s12325-021-01979-1.

R. Nishimura

Division of Diabetes, Metabolism and

Endocrinology, Department of Internal Medicine,

Jikei University School of Medicine, 3-25-8

Nishishinbashi, Minato-ku, Tokyo, Japan

M. Taniguchi $(\bowtie)$

Medical Affairs, Sumitomo Dainippon Pharma Co.,

Ltd., 1-13-1, Kyobashi, Chuo-ku, Tokyo, Japan

e-mail: mayumi-taniguchi@ds-pharma.co.jp

T. Takeshima $\cdot$ K. Iwasaki

Milliman Inc., 8F, Kojimachi 1-chome Building,

1-6-2 Kojimachi, Chiyoda-ku, Tokyo, Japan treatment in adult patients with T2D were selected by systematic review with comprehensive searching of CENTRAL, MEDLINE, Ichushi Web, and EMBASE and manual searching of clinical trial registries. The $\triangle \mathrm{HbA} 1 \mathrm{c}$ and incidence of hypoglycemia were compared among OAD treatments using Bayesian network metaanalysis (NMA). The relative risk (RR) of the incidence of hypoglycemia was determined relative to that of placebo.

Results: Forty-six randomized controlled trials were identified in the systematic review, and 37 studies, comprising 38 different types of treatments, including placebos, were selected for the NMA of $\triangle \mathrm{HbA} 1 \mathrm{c}$. Compared with metformin $1500 \mathrm{mg} /$ day, $20 \mathrm{OAD}$ treatments were significantly less effective in reducing $\mathrm{HbA1c}$ from baseline (differences from metformin $1500 \mathrm{mg}$ /day: $0.40-0.96 \%)$. Two treatments (glimepiride $2 \mathrm{mg} /$ day and pioglitazone $45 \mathrm{mg}$ /day) showed greater mean reductions in HbA1c from baseline than metformin $1500 \mathrm{mg} /$ day $(-0.38 \%$ and $-0.03 \%)$, although these differences were not significant. Regarding the incidence of hypoglycemia, only pioglitazone $30 \mathrm{mg} /$ day among 31 treatments showed a lower RR $(<-0.01)$, whereas 23 treatments showed a significantly higher RR (1.02-66.71) than metformin $1500 \mathrm{mg} /$ day.

Conclusion: The NMA suggested a preferable efficacy and safety profile of metformin $1500 \mathrm{mg} /$ day compared with the other OADs approved in Japan. 
Keywords: Glycated hemoglobin A; Hypoglycemia; Metformin; Network metaanalysis; Oral anti-diabetic drug; Systematic review; Type 2 diabetes

\section{Key Summary Points}

Why carry out this study?

No specific treatment is recommended, and various antidiabetic agents are used as first-line treatments for patients with type 2 diabetes (T2D) in Japan.

Metformin has long been used at low doses for patients with T2D in Japan, and information regarding its effects at the maximum maintenance dose is lacking.

We conducted a systematic review and network meta-analysis (NMA) to compare the efficacy and safety of metformin at $1500 \mathrm{mg} /$ day with those of the other oral anti-diabetes drugs (OADs) approved in Japan.

\section{What was learned from the study?}

Forty-six randomized controlled trials were identified in the systematic review, and 37 studies comprising 38 different types of treatments including placebos were selected for NMA of the reduction in hemoglobin A1c (HbA1c) from baseline.

Compared with metformin $1500 \mathrm{mg} /$ day, 20 treatments showed weak effects in reducing $\mathrm{HbA} 1 \mathrm{c}$ from baseline levels.

Metformin $1500 \mathrm{mg} /$ day showed preferable efficacy and safety profiles compared with the other OADs approved in Japan.

\section{INTRODUCTION}

Currently, metformin is recommended as a firstline glucose-lowering agent for patients with type 2 diabetes (T2D) in Western countries [1].
However, in Japan, no specific treatment is recommended, and the first-line treatment is recommended to be chosen after considering the pharmacological and adverse effect profiles to assess the disease condition for each patient [2]. Consequently, various agents including biguanide, thiazolidinediones, sulfonylurea, glinide, dipeptidyl peptidase-4 inhibitor (DPP$4 i), \alpha$-glucosidase inhibitor, and selective sodium-glucose transporter-2 inhibitor (SGLT2i) could be used as first-line treatments. Although the availability of these options may make their use preferable from the perspective of patient-centered care, physicians, particularly general practitioners, have difficulty selecting a treatment in real-world clinical settings. Therefore, information on the comparative efficacy of glucose-lowering agents is needed to select firstline treatment for Japanese patients with T2D.

Information regarding the effect of metformin at dosage levels similar to those used in Western countries compared with other treatments is lacking in Japan. Metformin has long been used at lower doses in Japan than in Western countries because of concerns related to the incidence of lactic acidosis caused by phenformin, an agent in the same biguanide class, in the 1970s [3]. Since 2010, the recommended dosage of metformin has been increased to $750-1500 \mathrm{mg} /$ day as the normal maintenance dose and $2250 \mathrm{mg}$ /day as the maximum prescription dose but the mean prescription dose typically remains below $1000 \mathrm{mg}$ /day in clinical settings in Japan $[4,5]$. Our internal survey on Japanese physicians showed that some Japanese physicians considered that the glycemic-lowering effect of metformin is lower than that of other treatments. A dose-dependent hypoglycemic effect of metformin compared with a placebo has been reported by clinical trials in Japan [3, 6]. However, the efficacy of metformin at doses similar to those used in Western countries in improving the hemoglobin A1c (HbA1c) level compared with other treatments approved in Japan has not been investigated in interventional or observational studies in Japanese patients with T2D. Such information could guide physicians in choosing first-line treatments. 
This study was conducted to compare the efficacy and safety of metformin at the maximum maintenance dose, $1500 \mathrm{mg} /$ day, with those of the other glucose-lowering agents in Japanese patients with T2D through a systematic review and network meta-analysis (NMA) of reported randomized controlled trials (RCTs). Considering that numerous first-line treatments are used in the Japanese clinical setting, NMA is a useful method for comparing treatments. We selected RCTs reporting results on the primary outcome of this study, that is, a change $(\Delta)$ in HbA1c from baseline at 12 weeks or later after the initiation of intervention. After summarizing the information of other outcomes reported in the included RCTs, we included the reduction rate of elevated $\mathrm{HbA} 1 \mathrm{c}$ and incidence rate of hypoglycemia as additional outcomes because of publicly available data for treatment with metformin at $1500 \mathrm{mg} /$ day.

\section{METHODS}

\section{Study Design}

A systematic review and an NMA were conducted to compare the efficacy and safety of metformin with those of the other oral anti-diabetes drugs (OADs) as first-line treatment in patients with T2D.

The study protocol was registered in PROSPERO (CRD42020160779). We followed the Cochrane Handbook for Systematic Reviews of Interventions [7] for the systematic review and the Preferred Reporting Items for Systematic Reviews and Meta-Analyses for Network MetaAnalysis (PRISMA-NMA) statement [8] to report the results. This study was based on previously conducted studies and does not involve any new studies with human participants or animals performed by any of the authors. Therefore, ethical approval and informed consent were not required.

\section{Eligibility Criteria}

The criteria for study inclusion in the systematic review and meta-analysis are summarized in
Table 1. Eligible studies were RCTs conducted in Japan that involved Japanese adult ( $\geq 20$ years) patients with $\mathrm{T} 2 \mathrm{D}$ and those either with no history of treatment with glucose-lowering agents or with a wash-out period before the RCT. Interventions in the RCTs were treatment for 12 weeks or longer with any OAD (from any class of biguanide, thiazolidinediones, sulfonylurea, glinide, DPP-4i, $\alpha$-glucosidase inhibitor, or SGLT2i) approved in Japan at the approved dosages. Comparators were another type of the abovementioned interventions (treatments with another type of OAD or a different dose of the same type of OAD) or a placebo for 12 weeks or longer. The primary outcome was the $\Delta$ in the HbA1c level from baseline at 12 weeks or later $(\Delta \mathrm{HbA} 1 \mathrm{c})$. The target publication period was the entire searchable period in each database described below.

\section{Search Strategy and Literature Review}

We comprehensively searched four databases: CENTRAL (Cochrane Library) on July 23, 2019, MEDLINE (via PubMed) on August 4, 2019, Ichushi Web (Japanese Medical Abstracts Society) on August 8, 2019, and EMBASE on November 29, 2019. The search terms included the disease name (T2D), names (generic names or compounds) or classes of OADs, study types, and languages. The search strategy for the CENTRAL database is shown in Supplementary Material Table S1. In addition, we searched three clinical trial registries: ClinicalTrials.gov, JAPIC Clinical Trials Information (JAPIC), and UMIN Clinical Trial Registry (UMIN) on May 13, 2020, to identify unpublished RCTs. The search terms applied to the manual search are shown in Supplementary Material Table S2.

We screened the identified RCTs to select eligible studies using the inclusion and exclusion criteria (Table 1). The inclusion criteria were (1) RCTs conducted in Japan, (2) patients and interventions/comparators met the eligibility criteria, (3) data of the primary outcome ( $\Delta$ HbA1c) were available, and (4) publication in English or Japanese. The exclusion criteria were (1) no information necessary for review (e.g., an abstract) available, (2) patients overlapped with 
those in other studies, (3) no data on the primary outcome, and (4) RCTs targeting patients with specified comorbidities. We included all publication types, including articles in peer-reviewed journals, conference abstracts, and data recorded in a clinical trials registry, if the RCTs were included in the target databases. If the measurement of $\Delta \mathrm{HbA} 1 \mathrm{c}$ at 12 weeks or later was described in the report but numerical data were not available, we acquired the study data from other data sources (e.g., clinical trial registry) or requested them from the corresponding authors. Among the studies in which patients overlapped, the study with data at or the closest to 12 weeks if studies included the same patient group or the study with the largest sample size if the studies included patient groups of different sizes was included. Patient overlap was identified based on the trial identification numbers or the following information in the reports: authors, location and settings, type of interventions, numbers and background information of the patients, and date and duration of RCTs. We included RCTs in any of the population types: intent-to-treat (ITT), full analysis set (FAS), or per-protocol set (PPS), and prioritized data from ITT or FAS population if data in multiple population types were provided. RCTs based on PPS population were excluded from the sensitivity analysis. Two reviewers separately selected studies using the following procedure: first, potential studies were extracted based on the abstract, and then the extracted studies were assessed based on the full text. Disagreements regarding study selection between the reviewers were resolved via a discussion.

\section{Data Extraction and Quality Assessment}

For data extraction, two reviewers independently screened the included RCTs. Each reviewer extracted the following data from the selected RCTs: basic information in the literature (authors, title, journal information [name of the journal, volume, issue, year, and pages]) or clinical trial (principal investigator, affiliation, and study year); study design; patient numbers (at baseline and evaluation); patient inclusion/exclusion criteria; intervention (drug type and dose); outcome data; length of the evaluation period; population type; and additional information if necessary.

An intervention was defined as a certain combination of drug type and dosage, and RCTs in which the intervention groups comprised patients with different final dosages were excluded. As additional outcomes, data on $\Delta$ high-density lipoprotein cholesterol, $\Delta$ lowdensity lipoprotein cholesterol, $\Delta$ total cholesterol, $\Delta$ non-high-density lipoprotein cholesterol, $\Delta$ triglyceride, $\Delta$ fasting plasma glucose, $\Delta$ glycoalbumin, $\Delta$ fasting serum insulin, and the incidence rate of hypoglycemia were extracted if the data were available.

As outcome data for the change at evaluation from baseline, information on the distribution of the mean value was extracted. If outcomes were evaluated at several time points at 12 weeks or later from the commencement of intervention, data at or the closest to 12 weeks were included. It is because 12 weeks from the commencement of intervention is considered to be the appropriate time when the change in HbA1c level stabilizes after the initiation of treatment and is the required time to evaluate treatment effects. We also considered that it is preferable to evaluate outcomes at the same period among treatments as much as possible, and 12 weeks was the most popular evaluation point among RCTs. If outcome data at 12 weeks were only graphically reported, without numerical values or distributions, we requested the data from the corresponding authors. If we did not obtain the numerical data at 12 weeks from the authors, we used the values at time points other than 12 weeks for which numerical values were reported. The incidence rate of hypoglycemia, reported as an independent outcome or adverse event, regardless of severity, was included. The data were recorded in an Excel spread sheet. When essential information could not be obtained, the RCTs were excluded. Disagreements on the extracted data between the reviewers were resolved via a discussion.

The risk of bias of the selected RCTs was assessed by two independent reviewers using the Revised Cochrane risk-of-bias tool for randomized trials [9]. Risk was evaluated for the 
primary outcome in each RCT. The risk for five domains was assessed: (1) bias arising from the randomization process, (2) bias due to deviations from the intended interventions, (3) bias due to missing outcome data, (4) bias in the measurement of the outcome, and (5) bias in the selection of the reported result. The overall risk of bias based on the risk of the domains was considered as a high risk, some concern, or low risk. The assessment results were subjected to sensitivity analysis to exclude RCTs with a high risk of bias. Disagreements between individual judgments were resolved via a discussion.

\section{Data Synthesis and Analysis}

Since April 1, 2012, the HbA1c level has been expressed as National Glycohemoglobin Standardization Program (NGSP) values rather than Japan Diabetes Society (JDS) values in Japan [10]. Therefore, the HbA1c levels reported as JDS values or likely reported as JDS values based on basic study information, such as study time and country, were converted to NGSP values by adding 0.4 to the JDS values [11]. We used the values of $\Delta$ in the mean HbA1c level as reported; if the values were not indicated in the studies, they were calculated from the mean HbA1c level at baseline and after intervention. If standard deviations (SDs) were not reported, they were calculated from the data distribution information, such as the $95 \%$ confidence interval and standard error (SE), if available.

As an additional outcome, we compared the HbA1c reduction rate using the cutoff value of $6.5 \%[2,12]$ for various OADs to reduce the effects of HbA1c differences at baseline, as the magnitude of HbA1c changes is reportedly associated with its baseline level [13]. That is, a higher baseline HbA1c level is associated with greater reductions. Assuming a model in which an OAD can reduce HbA1c level by a certain percentage, the reduction rate was calculated as $1-\frac{\text { HbA1c at evaluation }}{\text { HbA1c at baseline }}$. We considered the SD of the reduction rate of elevated HbA1c to be constant among interventions and calculated this value from the claims data provided by Medical Data Vision Co., Ltd. Among the additional outcomes extracted, those reported as results from treatment with $1500 \mathrm{mg} /$ day metformin were selected for NMA.

We prepared a network graph for each outcome for the main analysis or sensitivity analyses by the type of treatments consisting of drug types and doses. Treatments without a connection with any treatments were excluded from the NMA for each outcome or analysis.

The NMA was conducted using a Bayesian model with the Markov Chain Monte Carlo method. Two linear models were used to examine the effect of each treatment: a fixedeffect model written as a linear combination of the RCT and treatment effects for main analysis and a random-effect model expressing a linear combination of RCT, treatment, and random effects for sensitivity analysis. Prior probability distributions were assumed as flat likelihood functions using general(0) in the SAS MCMC procedure. For each outcome, the mean difference between treatment with metformin $1500 \mathrm{mg} /$ day and each of the other treatments was calculated, which were then assessed based on the difference in the posterior probability distributions with a two-tailed probability of 0.05 (probability of $<0.025$ or $>0.975$ ) considered as significant. We also calculated the mean difference between treatment with metformin $750 \mathrm{mg} /$ day and each of the other treatments to assess the difference in the effect depending on the dosage of metformin. As a Bayesian model was used, the consistency of the networks was reflected in the probabilistic distribution function of each variable. Subgroup analyses were not performed in this study.

We used SAS version 9.4 (SAS Institute, Cary, NC, USA) and Microsoft Excel 2016 (Microsoft Corporation, Redmond, WA, USA) for the analyses.

\section{Sensitivity Analysis}

We conducted three sensitivity analyses for $\Delta$ HbA1c. First, we applied a random-effects model to assess the model risk. Second, we excluded the RCTs considered as high risk based on the results of the risk of bias assessment. Finally, we excluded RCTs in which the outcomes were evaluated in a PPS population. 
Table 1 Criteria of study selection

Eligibility criteria

Patients

1. Japanese adults patients with T2D

2. Aged $\geq 20$ years at the RCT

3. Had no treatment history with glucose-lowering agents or had wash-out period before the RCT

Interventions and comparisons

Treatment with oral diabetic drugs or placebo for $\geq 12$ weeks

Primary outcome

Change in HbAlc from baseline

Study selection

Inclusion criteria

1. RCTs that conducted in Japan

2. Meeting patients and interventions/comparators to those in the eligibility criteria

3. Having data of primary outcome of the eligibility criteria

4. Published in English or Japanese

Exclusion criteria

1. Without necessary information for literature review by the search of databases

2. Overlapped the participants with other studies

3. Without data of primary outcome of the eligibility criteria

4. Targeted patients with specified comorbidities

$H b A 1 c$ hemoglobin A1c, $O A D$ oral anti-diabetes drug, $R C T$ randomized controlled trial, $T 2 D$ type 2 diabetes

\section{RESULTS}

\section{Study Selection and Characteristics}

A literature search identified 2375 studies from four databases, including 1556 unique studies, which were assessed for relevance (Fig. 1). Fortyfive RCTs, including one RCT from a clinical trials registry database included in CENTRAL [14] and the others from articles in peer-reviewed journals, were selected (Fig. 1). The number of RCTs by reasons for exclusion is shown in Supplementary Material Table S3. Manual searching retrieved 98, 268, and 152 RCTs from ClinicalTrials.gov, JAPIC, and UMIN, respectively, using the search terms described in Supplementary Material Table S2, of which 9, 14 , and 4 RCTs met or were likely to meet the eligibility criteria. After assessing the information in detail, all RCTs from ClinicalTrials.gov were found to be duplicates of RCTs already identified in the previous literature search; from JAPIC, three RCTs did not meet the criteria, ten RCTs were already included, and one RCT [15] was selected as eligible. Among those from UMIN, one RCT was already included and three did not show the results (Fig. 1).

In the 46 RCTs, fasting plasma glucose was the most frequently reported additional outcome (in 40 RCTs), followed by the hypoglycemia incidence rate (36 RCTs). The number 


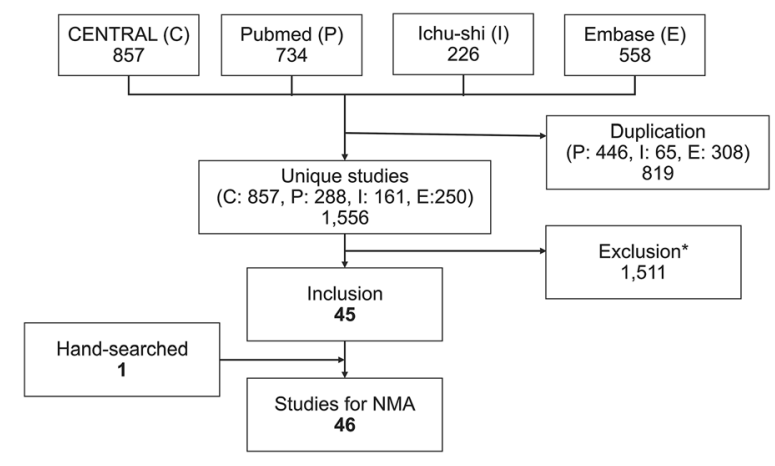

Fig. 1 Flow diagram of study selection. ${ }^{*}$ Note: number of randomized controlled trials by reasons for exclusion is shown in Supplementary Material Table S3. NMA: network meta-analysis.

of RCTs reporting each additional outcome is shown in Supplementary Material Table S4 with detailed literature information for each RCT. In one RCT, treatment with metformin at $1500 \mathrm{mg} /$ day was included as an intervention, and the outcomes reported were $\Delta \mathrm{HbA} 1 \mathrm{c}$ with baseline HbA1c and the incidence rate of hypoglycemia [15]. Therefore, we analyzed the reduction rate of elevated $\mathrm{HbA} 1 \mathrm{c}$ and incidence rate of hypoglycemia as additional outcomes. Outcome data of each study are summarized in Supplementary Material Table S5.

Basic information on the RCTs obtained through data extraction is shown in Table 2. The treatment duration until $\Delta$ HbA1c evaluation extracted for the NMA was 12 weeks for 32 RCTs, 14 weeks for 1 RCT, 16 weeks for 1 RCT, 24 weeks for 11 RCTs, and 28 weeks for 1 RCT (Table 2). Among 14 RCTs without data at 12 weeks, we attempted to contact the corresponding authors or co-authors of 8 RCTs. However, we could not obtain the data because the authors of three RCTs responded that they do not have the data, and the others did not respond. We did not contact authors of six RCTs as the contact information was not available (for three RCTs) or we thought that they likely did not record the data at 12 weeks based on the protocol or the methods in the manuscript (for three RCTs). We excluded RCTs targeting patients with comorbidities (e.g., chronic kidney disease, chronic heart failure, coronary artery disease, and non-alcoholic fatty liver disease), whereas those of patients with metabolic syndrome $[16,17]$ or overweight $[18]$ were included. Eight RCTs [19-26] evaluated the primary outcome in a PPS or a similar-to-PPS.

Through a risk of bias assessment to determine the overall risk of bias, 10 RCTs $[18-21,23,24,26,34,48,50]$ were considered high risk and 4 and 32 RCTs were considered low risk and some concern, respectively (Supplementary Material Fig. S1). The high-risk RCTs included six RCTs in PPS or similar-to-PPS [19-21, 23, 24, 26], two RCTs published in the 1990s [21, 26], one RCT with a cross-over design [50], two RCTs with an open-label design $[18,19]$, and one RCT in patients who were overweight [18].

Among the selected RCTs, those without SD data for the mean $\triangle \mathrm{HbA} 1 \mathrm{c}$ or other information to calculate the SD, including the SE or $95 \%$ confidence interval, were excluded from the $\triangle \mathrm{HbA} 1 \mathrm{c}$ analysis. In the analysis of the reduction rate of elevated $\mathrm{HbA1c}$, RCTs without baseline HbA1c level and those with baseline $\mathrm{HbA1c}$ at $<6.5 \%$ were excluded. As a result, one RCT [37] was not included in the analysis of any outcome. The number of RCTs finally included in the analysis of each outcome, that is, $\triangle \mathrm{HbA} 1 \mathrm{c}$, reduction rate of elevated $\mathrm{HbA} 1 \mathrm{c}$, and incidence rate of hypoglycemia, was 37,42 , and 36, respectively, which included 96, 106, and 92 treatment arms, respectively (Table 2). Among the RCTs included for each outcome, 38, 38, and 33 different types of treatments (including placebo), respectively, were included.

Treatments assessed for the primary outcome were one of the two biguanide drugs approved in Japan (metformin 750 or $1500 \mathrm{mg}$ /day), one thiazolidinedione drug (pioglitazone 15, 30, or $45 \mathrm{mg} /$ day), one of the six sulfonylurea drugs (glimepiride $2 \mathrm{mg} /$ day), all three glinide drugs (nateglinide $270 \mathrm{mg} /$ day, mitiglinide 30 or $60 \mathrm{mg} /$ day, and repaglinide $0.75,1.5$, or $3 \mathrm{mg} /$ day), all nine DPP-4i drugs (sitagliptin 100 or $50 \mathrm{mg} /$ day, vildagliptin $100 \mathrm{mg} /$ day, alogliptin $25 \mathrm{mg} /$ day, linagliptin $5 \mathrm{mg} /$ day, teneligliptin 20 and $40 \mathrm{mg} /$ day, anagliptin 200 or $400 \mathrm{mg} /$ day, saxagliptin $5 \mathrm{mg} /$ day, trelagliptin $100 \mathrm{mg} /$ week, and omarigliptin $25 \mathrm{mg} /$ week), two of the three $\alpha$-glucosidase inhibitor drugs (voglibose 0.6 and $0.9 \mathrm{mg} / \mathrm{day}$, and miglitol 
Table 2 Basic characteristics of the identified RCTs

\begin{tabular}{|c|c|c|c|c|c|c|c|}
\hline \multirow[t]{2}{*}{ ID } & \multirow[t]{2}{*}{ Study } & \multirow{2}{*}{$\begin{array}{l}\text { Treatment } \\
\text { duration }^{a} \\
\text { (weeks) }\end{array}$} & \multirow{2}{*}{$\begin{array}{l}\text { Treatment } \\
\text { (drug generic } \\
\text { name, dosage) }\end{array}$} & \multirow{2}{*}{$\begin{array}{l}\text { Patient } \\
\text { number }\end{array}$} & \multicolumn{3}{|c|}{ Being included/excluded for NMA } \\
\hline & & & & & $\begin{array}{l}\Delta \\
\text { HbAlc }\end{array}$ & $\begin{array}{l}\text { Reduction rate of } \\
\text { elevated } \mathrm{HbAlc}\end{array}$ & Hypoglycemia \\
\hline \multirow[t]{2}{*}{39} & \multirow[t]{2}{*}{$\begin{array}{l}\text { Tsurutani } \\
2018[19]\end{array}$} & \multirow[t]{2}{*}{12} & $\begin{array}{l}\text { Sitagliptin } \\
50 \mathrm{mg} / \text { day }\end{array}$ & 49 & \multirow[t]{2}{*}{ Included } & \multirow[t]{2}{*}{ Excluded } & \multirow[t]{2}{*}{ Included } \\
\hline & & & $\begin{array}{l}\text { Ipragliflozin } \\
50 \mathrm{mg} / \text { day }\end{array}$ & 52 & & & \\
\hline \multirow[t]{2}{*}{54} & \multirow{2}{*}{$\begin{array}{l}\text { Yamada } \\
2018 \text { [27] }\end{array}$} & \multirow[t]{2}{*}{12} & placebo & 72 & \multirow[t]{2}{*}{ Excluded } & \multirow[t]{2}{*}{ Included } & \multirow[t]{2}{*}{ Included } \\
\hline & & & $\begin{array}{l}\text { Sitagliptin } \\
50 \mathrm{mg} / \text { day }\end{array}$ & 75 & & & \\
\hline \multirow[t]{4}{*}{76} & \multirow{4}{*}{$\begin{array}{l}\text { Kaku 2012a } \\
\quad[28]\end{array}$} & \multirow[t]{4}{*}{12} & placebo & 58 & \multirow[t]{4}{*}{ Included } & \multirow[t]{4}{*}{ Included } & \multirow[t]{4}{*}{ Included } \\
\hline & & & $\begin{array}{l}\text { Anagliptin } \\
200 \mathrm{mg} / \text { day }\end{array}$ & 63 & & & \\
\hline & & & $\begin{array}{l}\text { Anagliptin } \\
\qquad 400 \mathrm{mg} / \text { day }\end{array}$ & 58 & & & \\
\hline & & & $\begin{array}{l}\text { Voglibose } \\
0.6 \mathrm{mg} / \text { day }\end{array}$ & 65 & & & \\
\hline \multirow[t]{3}{*}{77} & \multirow{3}{*}{$\begin{array}{l}\text { Kaku } \\
\text { 2012b } \\
{[20]}\end{array}$} & \multirow[t]{3}{*}{12} & placebo & 63 & \multirow[t]{3}{*}{ Included } & \multirow[t]{3}{*}{ Included } & \multirow[t]{3}{*}{ Included } \\
\hline & & & $\begin{array}{l}\text { Anagliptin } \\
200 \mathrm{mg} / \text { day }\end{array}$ & 69 & & & \\
\hline & & & $\begin{array}{l}\text { Anagliptin } \\
\qquad 400 \mathrm{mg} / \text { day }\end{array}$ & 68 & & & \\
\hline \multirow[t]{3}{*}{93} & \multirow{3}{*}{$\begin{array}{l}\text { Seino } \\
\qquad 2014 \mathrm{a} \\
{[29]}\end{array}$} & \multirow[t]{3}{*}{12} & placebo & 87 & \multirow[t]{3}{*}{ Included } & Included & Included \\
\hline & & & Saxagliptin & 82 & & & \\
\hline & & & $5 \mathrm{mg} /$ day & & & & \\
\hline 100 & Kashiwagi & 16 & placebo & 67 & Included & Included & Included \\
\hline & $2015[30]$ & & $\begin{array}{l}\text { Ipragliflozin } \\
50 \mathrm{mg} / \mathrm{day}\end{array}$ & 62 & & & \\
\hline 256 & Kaku $2014 a$ & 24 & placebo & 87 & Included & Included & Included \\
\hline & {$[31]$} & & $\begin{array}{c}\text { Dapagliflozin } \\
5 \mathrm{mg} / \text { day }\end{array}$ & 86 & & & \\
\hline & & & $\begin{array}{c}\text { Dapagliflozin } \\
10 \mathrm{mg} / \text { day }\end{array}$ & 88 & & & \\
\hline 258 & $\begin{array}{l}\text { Kaku } \\
\text { 2014b } \\
{[32]}\end{array}$ & 24 & placebo & 56 & Included & Included & Included \\
\hline
\end{tabular}


Table 2 contiuned

\begin{tabular}{|c|c|c|c|c|c|c|c|}
\hline \multirow[t]{2}{*}{ ID } & \multirow[t]{2}{*}{ Study } & \multirow{2}{*}{$\begin{array}{l}\text { Treatment } \\
\text { duration }^{a} \\
\text { (weeks) }\end{array}$} & \multirow{2}{*}{$\begin{array}{l}\text { Treatment } \\
\text { (drug generic } \\
\text { name, dosage) }\end{array}$} & \multirow{2}{*}{$\begin{array}{l}\text { Patient } \\
\text { number }\end{array}$} & \multicolumn{3}{|c|}{ Being included/excluded for NMA } \\
\hline & & & & & $\begin{array}{l}\Delta \\
\text { HbAlc }\end{array}$ & $\begin{array}{l}\text { Reduction rate of } \\
\text { elevated } \mathrm{HbAlc}\end{array}$ & Hypoglycemia \\
\hline & & & $\begin{array}{l}\text { Tofogliflozin } \\
20 \mathrm{mg} / \text { day }\end{array}$ & 58 & & & \\
\hline \multirow[t]{5}{*}{282} & Kadowaki & 12 & placebo & 109 & Included & Included & Included \\
\hline & 2014 [33] & & Empagliflozin & 109 & & & \\
\hline & & & $10 \mathrm{mg} /$ day & & & & \\
\hline & & & Empagliflozin & 109 & & & \\
\hline & & & $25 \mathrm{mg} /$ day & & & & \\
\hline \multirow[t]{4}{*}{296} & Fujitani & 12 & Linagliptin & 188 & Included & Included & Included \\
\hline & 2016 [34] & & $5 \mathrm{mg} /$ day & & & & \\
\hline & & & Voglibose & 178 & & & \\
\hline & & & $0.6 \mathrm{mg} / \mathrm{day}$ & & & & \\
\hline \multirow[t]{5}{*}{348} & Gantz 2017 & 24 & placebo & 82 & Included & Included & Included \\
\hline & {$[35]$} & & Sitagliptin & 164 & & & \\
\hline & & & $50 \mathrm{mg} /$ day & & & & \\
\hline & & & Omarigliptin & 166 & & & \\
\hline & & & $25 \mathrm{mg} /$ week & & & & \\
\hline \multirow[t]{4}{*}{360} & Satoh 2017 & 12 & Linagliptin & 47 & Included & Included & $\mathrm{N} / \mathrm{A}$ \\
\hline & {$[36]$} & & $5 \mathrm{mg} /$ day & & & & \\
\hline & & & Voglibose & 52 & & & \\
\hline & & & $0.6 \mathrm{mg} /$ day & & & & \\
\hline \multirow[t]{3}{*}{366} & Hotta 1993 & 24 & placebo & 13 & Excluded & Included & $\mathrm{N} / \mathrm{A}$ \\
\hline & {$[21]$} & & Acarbose & 16 & & & \\
\hline & & & $300 \mathrm{mg} /$ day & & & & \\
\hline \multirow[t]{4}{*}{380} & Kato 2009 & 12 & Metformin & 25 & Excluded & Included & $\mathrm{N} / \mathrm{A}$ \\
\hline & {$[16]$} & & $500 \mathrm{mg} /$ day & & & & \\
\hline & & & Pioglitazone & 25 & & & \\
\hline & & & $15 \mathrm{mg} /$ day & & & & \\
\hline \multirow[t]{4}{*}{387} & Kato 2010 & 12 & Nateglinide & 10 & Excluded & Excluded & $\mathrm{N} / \mathrm{A}$ \\
\hline & {$[37]$} & & $270 \mathrm{mg} /$ day & & & & \\
\hline & & & Acarbose & 10 & & & \\
\hline & & & 300 mg/day & & & & \\
\hline
\end{tabular}


Table 2 contiuned

\begin{tabular}{|c|c|c|c|c|c|c|c|}
\hline \multirow[t]{2}{*}{ ID } & \multirow[t]{2}{*}{ Study } & \multirow{2}{*}{$\begin{array}{l}\text { Treatment } \\
\text { duration }^{\mathrm{a}} \\
\text { (weeks) }\end{array}$} & \multirow{2}{*}{$\begin{array}{l}\text { Treatment } \\
\text { (drug generic } \\
\text { name, dosage) }\end{array}$} & \multirow{2}{*}{$\begin{array}{l}\text { Patient } \\
\text { number }\end{array}$} & \multicolumn{3}{|c|}{ Being included/excluded for NMA } \\
\hline & & & & & $\begin{array}{l}\Delta \\
\text { HbAlc }\end{array}$ & $\begin{array}{l}\text { Reduction rate of } \\
\text { elevated HbA1c }\end{array}$ & Hypoglycemia \\
\hline \multirow[t]{3}{*}{391} & \multirow{3}{*}{$\begin{array}{c}\text { Iwamoto } \\
\text { 2010a } \\
{[38]}\end{array}$} & \multirow[t]{3}{*}{12} & placebo & 73 & \multirow[t]{3}{*}{ Included } & \multirow[t]{3}{*}{ Included } & \multirow[t]{3}{*}{ Included } \\
\hline & & & $\begin{array}{l}\text { Sitagliptin } \\
\quad 50 \mathrm{mg} / \text { day }\end{array}$ & 72 & & & \\
\hline & & & $\begin{array}{l}\text { Sitagliptin } \\
100 \mathrm{mg} / \text { day }\end{array}$ & 70 & & & \\
\hline \multirow[t]{2}{*}{393} & \multirow{2}{*}{$\begin{array}{c}\text { Iwamoto } \\
2010 \mathrm{~b} \\
{[22]}\end{array}$} & \multirow[t]{2}{*}{12} & $\begin{array}{l}\text { Sitagliptin } \\
50 \mathrm{mg} / \text { day }\end{array}$ & 155 & \multirow[t]{2}{*}{ Included } & \multirow[t]{2}{*}{ Included } & \multirow[t]{2}{*}{ Included } \\
\hline & & & $\begin{array}{l}\text { Voglibose } \\
0.6 \mathrm{mg} / \text { day }\end{array}$ & 146 & & & \\
\hline \multirow[t]{2}{*}{406} & \multirow{2}{*}{$\begin{array}{c}\text { Iwamoto } \\
\text { 2010c } \\
{[39]}\end{array}$} & \multirow[t]{2}{*}{12} & $\begin{array}{l}\text { Vildagliptin } \\
100 \mathrm{mg} / \text { day }\end{array}$ & 188 & \multirow[t]{2}{*}{ Excluded } & \multirow[t]{2}{*}{ Included } & \multirow[t]{2}{*}{ Included } \\
\hline & & & $\begin{array}{l}\text { Voglibose } \\
0.6 \mathrm{mg} / \text { day }\end{array}$ & 192 & & & \\
\hline \multirow[t]{3}{*}{417} & \multirow{3}{*}{$\begin{array}{l}\text { Seino } 2011 \\
\quad[40]\end{array}$} & \multirow[t]{3}{*}{12} & placebo & 75 & \multirow[t]{3}{*}{ Included } & \multirow[t]{3}{*}{ Included } & \multirow[t]{3}{*}{ Included } \\
\hline & & & $\begin{array}{l}\text { Alogliptin } \\
25 \mathrm{mg} / \text { day }\end{array}$ & 80 & & & \\
\hline & & & $\begin{array}{l}\text { Voglibose } \\
0.6 \mathrm{mg} / \text { day }\end{array}$ & 83 & & & \\
\hline \multirow[t]{3}{*}{426} & \multirow{3}{*}{$\begin{array}{l}\text { Kawamori } \\
2012[41]\end{array}$} & \multirow[t]{3}{*}{12} & placebo & 80 & \multirow[t]{3}{*}{ Included } & \multirow[t]{3}{*}{ Included } & Included \\
\hline & & & $\begin{array}{l}\text { Linagliptin } \\
5 \mathrm{mg} / \text { day }\end{array}$ & 159 & & & \\
\hline & & & $\begin{array}{l}\text { Voglibose } \\
0.6 \mathrm{mg} / \text { day }\end{array}$ & 162 & & & \\
\hline 443 & Inagaki & 12 & placebo & 75 & Excluded & Included & Included \\
\hline & $2013[42]$ & & $\begin{array}{l}\text { Canagliflozin } \\
100 \mathrm{mg} / \text { day }\end{array}$ & 74 & & & \\
\hline 445 & Kadowaki & 12 & placebo & 80 & Included & Included & Included \\
\hline & $2013[43]$ & & $\begin{array}{r}\text { Teneligliptin } \\
20 \mathrm{mg} / \text { day }\end{array}$ & 79 & & & \\
\hline & & & $\begin{array}{r}\text { Teneligliptin } \\
40 \mathrm{mg} / \text { day }\end{array}$ & 81 & & & \\
\hline
\end{tabular}


Table 2 contiuned

\begin{tabular}{|c|c|c|c|c|c|c|c|}
\hline \multirow[t]{2}{*}{ ID } & \multirow[t]{2}{*}{ Study } & \multirow{2}{*}{$\begin{array}{l}\text { Treatment } \\
\text { duration }^{a} \\
\text { (weeks) }\end{array}$} & \multirow{2}{*}{$\begin{array}{l}\text { Treatment } \\
\text { (drug generic } \\
\text { name, dosage) }\end{array}$} & \multirow{2}{*}{$\begin{array}{l}\text { Patient } \\
\text { number }\end{array}$} & \multicolumn{3}{|c|}{ Being included/excluded for NMA } \\
\hline & & & & & $\begin{array}{l}\Delta \\
\text { HbAlc }\end{array}$ & $\begin{array}{l}\text { Reduction rate of } \\
\text { elevated HbAlc }\end{array}$ & Hypoglycemia \\
\hline \multirow[t]{3}{*}{453} & \multirow{3}{*}{$\begin{array}{l}\text { Inagaki } \\
\text { 2014a [44] }\end{array}$} & \multirow[t]{3}{*}{12} & placebo & 55 & \multirow[t]{3}{*}{ Included } & \multirow[t]{3}{*}{ Included } & \multirow[t]{3}{*}{ Included } \\
\hline & & & Trelagliptin & 55 & & & \\
\hline & & & $100 \mathrm{mg} /$ week & & & & \\
\hline \multirow[t]{3}{*}{460} & \multirow{3}{*}{$\begin{array}{l}\text { Seino } 2014 b \\
\quad[45]\end{array}$} & \multirow[t]{3}{*}{24} & placebo & 79 & \multirow[t]{3}{*}{ Included } & \multirow[t]{3}{*}{ Included } & \multirow[t]{3}{*}{ Included } \\
\hline & & & Luseogliflozin & 79 & & & \\
\hline & & & $2.5 \mathrm{mg} /$ day & & & & \\
\hline \multirow[t]{5}{*}{461} & \multirow{5}{*}{$\begin{array}{l}\text { Seino } 2014 c \\
\quad[46]\end{array}$} & \multirow[t]{5}{*}{12} & placebo & 57 & \multirow[t]{5}{*}{ Included } & \multirow[t]{5}{*}{ Included } & \multirow[t]{5}{*}{ Included } \\
\hline & & & Luseogliflozin & 56 & & & \\
\hline & & & $2.5 \mathrm{mg} /$ day & & & & \\
\hline & & & Luseogliflozin & 54 & & & \\
\hline & & & $5 \mathrm{mg} /$ day & & & & \\
\hline \multirow[t]{5}{*}{462} & \multirow{5}{*}{$\begin{array}{l}\text { Seino } 2014 \mathrm{~d} \\
\quad[47]\end{array}$} & \multirow[t]{5}{*}{12} & placebo & 54 & \multirow[t]{5}{*}{ Included } & Included & Included \\
\hline & & & Luseogliflozin & 61 & & & \\
\hline & & & $2.5 \mathrm{mg} / \mathrm{day}$ & & & & \\
\hline & & & Luseogliflozin & 61 & & & \\
\hline & & & $5 \mathrm{mg} /$ day & & & & \\
\hline 467 & Inagaki & 24 & placebo & 93 & Included & Included & Included \\
\hline & $2014 b[48]$ & & Canagliflozin & 90 & & & \\
\hline & & & $100 \mathrm{mg} /$ day & & & & \\
\hline 474 & Mikada 2014 & 24 & Sitagliptin & 14 & Included & Included & $\mathrm{N} / \mathrm{A}$ \\
\hline & {$[18]$} & & $50 \mathrm{mg} /$ day & & & & \\
\hline & & & Miglitol & 14 & & & \\
\hline & & & $150 \mathrm{mg} /$ day & & & & \\
\hline 484 & Inagaki 2015 & 24 & placebo & 50 & Included & Included & Included \\
\hline & [49] & & Alogliptin & 92 & & & \\
\hline & & & $25 \mathrm{mg} /$ day & & & & \\
\hline & & & Trelagliptin & 101 & & & \\
\hline & & & $100 \mathrm{mg} /$ week & & & & \\
\hline 521 & Kurebayashi & 12 & Nateglinide & 14 & Excluded & Included & $\mathrm{N} / \mathrm{A}$ \\
\hline & $2006[50]$ & & $270 \mathrm{mg} /$ day & & & & \\
\hline & & & Voglibose & 15 & & & \\
\hline & & & $0.6 \mathrm{mg} /$ day & & & & \\
\hline
\end{tabular}


Table 2 contiuned

\begin{tabular}{|c|c|c|c|c|c|c|c|}
\hline \multirow[t]{2}{*}{ ID } & \multirow[t]{2}{*}{ Study } & \multirow{2}{*}{$\begin{array}{l}\text { Treatment } \\
\text { duration }^{a} \\
\text { (weeks) }\end{array}$} & \multirow{2}{*}{$\begin{array}{l}\text { Treatment } \\
\text { (drug generic } \\
\text { name, } \\
\text { dosage) }\end{array}$} & \multirow{2}{*}{$\begin{array}{l}\text { Patient } \\
\text { number }\end{array}$} & \multicolumn{3}{|c|}{ Being included/excluded for NMA } \\
\hline & & & & & $\begin{array}{l}\Delta \\
\text { HbAlc }\end{array}$ & $\begin{array}{l}\text { Reduction rate of } \\
\text { elevated } \mathrm{HbAlc}\end{array}$ & Hypoglycemia \\
\hline \multirow[t]{2}{*}{539} & \multirow{2}{*}{$\begin{array}{l}\text { Kikuchi } 2009 \\
\text { [51] }\end{array}$} & \multirow[t]{2}{*}{12} & placebo & 72 & \multirow[t]{2}{*}{ Excluded } & \multirow[t]{2}{*}{ Included } & \multirow[t]{2}{*}{ Included } \\
\hline & & & $\begin{array}{l}\text { Vildagliptin } \\
100 \mathrm{mg} / \text { day }\end{array}$ & 76 & & & \\
\hline \multirow[t]{2}{*}{541} & \multirow[t]{2}{*}{$\begin{array}{l}\text { Yokoyama } 2009 \\
\text { [23] }\end{array}$} & \multirow[t]{2}{*}{$12^{\mathrm{a}}$} & $\begin{array}{l}\text { Mitiglinide } \\
\quad 30 \mathrm{mg} / \text { day }\end{array}$ & 17 & \multirow[t]{2}{*}{ Excluded } & \multirow[t]{2}{*}{ Included } & \multirow[t]{2}{*}{ No data } \\
\hline & & & $\begin{array}{l}\text { Miglitol } \\
150 \mathrm{mg} / \text { day }\end{array}$ & 19 & & & \\
\hline \multirow[t]{2}{*}{545} & \multirow{2}{*}{$\begin{array}{l}\text { Nonaka } 2008 \\
\text { [52] }\end{array}$} & \multirow[t]{2}{*}{12} & placebo & 75 & \multirow[t]{2}{*}{ Included } & \multirow[t]{2}{*}{ Included } & \multirow[t]{2}{*}{ Included } \\
\hline & & & $\begin{array}{l}\text { Sitagliptin } \\
\qquad 100 \mathrm{mg} / \text { day }\end{array}$ & 75 & & & \\
\hline \multirow[t]{2}{*}{597} & \multirow{2}{*}{$\begin{array}{l}\text { NCT00998881 } \\
\quad[14]\end{array}$} & \multirow[t]{2}{*}{12} & placebo & 104 & \multirow[t]{2}{*}{ Included } & \multirow[t]{2}{*}{ Excluded } & \multirow[t]{2}{*}{ Included } \\
\hline & & & $\begin{array}{l}\text { Teneligliptin } \\
20 \mathrm{mg} / \text { day }\end{array}$ & 99 & & & \\
\hline \multirow[t]{3}{*}{682} & \multirow{3}{*}{$\begin{array}{l}\text { Kashiwagi } 2014 \\
\quad[53]\end{array}$} & \multirow[t]{3}{*}{12} & placebo & 69 & \multirow[t]{3}{*}{ Included } & Included & Included \\
\hline & & & $\begin{array}{l}\text { Ipragliflozin } \\
50 \mathrm{mg} / \text { day }\end{array}$ & 72 & & & \\
\hline & & & $\begin{array}{l}\text { Ipragliflozin } \\
100 \mathrm{mg} / \text { day }\end{array}$ & 72 & & & \\
\hline 742 & Tanaka 2007 & 12 & placebo & 40 & Included & Included & Included \\
\hline & {$[24]$} & & $\begin{array}{l}\text { Mitiglinide } \\
30 \mathrm{mg} / \text { day }\end{array}$ & 45 & & & \\
\hline & & & $\begin{array}{l}\text { Mitiglinide } \\
\qquad 60 \mathrm{mg} / \text { day }\end{array}$ & 43 & & & \\
\hline 748 & Kaneko 1997 & 12 & placebo & 66 & Included & Included & Included \\
\hline & [54] & & $\begin{array}{l}\text { Pioglitazone } \\
30 \mathrm{mg} / \text { day }\end{array}$ & 68 & & & \\
\hline $\mathrm{I}-135$ & Koh 2010 [55] & 24 & $\begin{array}{l}\text { Glimepiride } \\
2 \mathrm{mg} / \text { day }\end{array}$ & 47 & Included & Excluded & Excluded \\
\hline & & & $\begin{array}{l}\text { Voglibose } \\
0.9 \mathrm{mg} / \text { day }\end{array}$ & 49 & & & \\
\hline $\mathrm{I}-138$ & Kikuchi 2010 & 12 & placebo & 59 & Included & Included & Included \\
\hline & {$[56]$} & & $\begin{array}{l}\text { Vildagliptin } \\
100 \mathrm{mg} / \text { day }\end{array}$ & 63 & & & \\
\hline
\end{tabular}


Table 2 contiuned

\begin{tabular}{|c|c|c|c|c|c|c|c|}
\hline \multirow[t]{2}{*}{ ID } & \multirow[t]{2}{*}{ Study } & \multirow{2}{*}{$\begin{array}{l}\text { Treatment } \\
\text { duration }^{a} \\
\text { (weeks) }\end{array}$} & \multirow{2}{*}{$\begin{array}{l}\text { Treatment } \\
\text { (drug generic } \\
\text { name, } \\
\text { dosage) }\end{array}$} & \multirow{2}{*}{$\begin{array}{l}\text { Patient } \\
\text { number }\end{array}$} & \multicolumn{3}{|c|}{ Being included/excluded for NMA } \\
\hline & & & & & $\begin{array}{l}\Delta \\
\text { HbAlc }\end{array}$ & $\begin{array}{l}\text { Reduction rate of } \\
\text { elevated HbAlc }\end{array}$ & Hypoglycemia \\
\hline \multirow[t]{2}{*}{$\mathrm{I}-204$} & $\begin{array}{l}\text { Kosaka } 1997 \\
\quad[25]\end{array}$ & 12 & $\begin{array}{l}\text { Nateglinide } \\
270 \mathrm{mg} / \text { day }\end{array}$ & 83 & Included & Included & Included \\
\hline & & & $\begin{array}{l}\text { Voglibose } \\
0.6 \mathrm{mg} / \text { day }\end{array}$ & 77 & & & \\
\hline \multirow[t]{4}{*}{$\mathrm{I}-209$} & Kaneko 1997 & 12 & placebo & 60 & Included & Included & $\mathrm{N} / \mathrm{A}$ \\
\hline & {$[26]$} & & $\begin{array}{l}\text { Pioglitazone } \\
15 \mathrm{mg} / \text { day }\end{array}$ & 63 & & & \\
\hline & & & $\begin{array}{l}\text { Pioglitazone } \\
30 \mathrm{mg} / \text { day }\end{array}$ & 57 & & & \\
\hline & & & $\begin{array}{l}\text { Pioglitazone } \\
45 \mathrm{mg} / \text { day }\end{array}$ & 54 & & & \\
\hline \multirow[t]{2}{*}{$\mathrm{I}-217$} & Kamiya 1992 & 28 & placebo & 44 & Included & Included & $\mathrm{N} / \mathrm{A}$ \\
\hline & {$[57]$} & & $\begin{array}{l}\text { Voglibose } \\
0.6 \mathrm{mg} / \text { day }\end{array}$ & 42 & & & \\
\hline \multirow[t]{4}{*}{$\mathrm{I}-71$} & Koiwai 2015 & 24 & placebo & 41 & Included & Included & Included \\
\hline & {$[58]$} & & $\begin{array}{l}\text { Sitagliptin } \\
100 \mathrm{mg} / \text { day }\end{array}$ & 41 & & & \\
\hline & & & $\begin{array}{l}\text { Empagliflozin } \\
10 \mathrm{mg} / \text { day }\end{array}$ & 43 & & & \\
\hline & & & $\begin{array}{c}\text { Empagliflozin } \\
25 \mathrm{mg} / \text { day }\end{array}$ & 43 & & & \\
\hline \multirow[t]{4}{*}{$\mathrm{I}-92$} & Kawamori2014 & 12 & placebo & 36 & Included & Included & Included \\
\hline & [59] & & $\begin{array}{l}\text { Repaglinide } \\
0.75 \mathrm{mg} / \text { day }\end{array}$ & 37 & & & \\
\hline & & & $\begin{array}{l}\text { Repaglinide } \\
1.5 \mathrm{mg} / \text { day }\end{array}$ & 36 & & & \\
\hline & & & $\begin{array}{r}\text { Repaglinide } \\
3 \mathrm{mg} / \text { day }\end{array}$ & 37 & & & \\
\hline \multirow[t]{2}{*}{ P-501 } & $\begin{array}{l}\text { Fujitaka } 2011 \\
\quad[17]\end{array}$ & 24 & $\begin{array}{l}\text { Voglibose } \\
0.9 \mathrm{mg} / \text { day }\end{array}$ & 30 & Included & Excluded & $\mathrm{N} / \mathrm{A}$ \\
\hline & & & $\begin{array}{l}\text { Pioglitazone } \\
30 \mathrm{mg} / \text { day }\end{array}$ & 30 & & & \\
\hline
\end{tabular}


Table 2 contiuned

\begin{tabular}{|c|c|c|c|c|c|c|c|}
\hline \multirow[t]{2}{*}{ ID } & \multirow[t]{2}{*}{ Study } & \multirow{2}{*}{$\begin{array}{l}\text { Treatment } \\
\text { duration }^{\mathrm{a}} \\
\text { (weeks) }\end{array}$} & \multirow{2}{*}{$\begin{array}{l}\text { Treatment } \\
\text { (drug generic } \\
\text { name, } \\
\text { dosage) }\end{array}$} & \multirow{2}{*}{$\begin{array}{l}\text { Patient } \\
\text { number }\end{array}$} & \multicolumn{3}{|c|}{ Being included/excluded for NMA } \\
\hline & & & & & $\begin{array}{l}\Delta \\
\text { HbAlc }\end{array}$ & $\begin{array}{l}\text { Reduction rate of } \\
\text { elevated HbA1c }\end{array}$ & Hypoglycemia \\
\hline \multirow{3}{*}{$\begin{array}{l}\text { Hand- } \\
\text { search }\end{array}$} & \multirow{3}{*}{$\begin{array}{c}\text { JapicCTI- } \\
050090 \\
{[15]}\end{array}$} & \multirow[t]{3}{*}{14} & placebo & 55 & \multirow[t]{3}{*}{ Included } & \multirow[t]{3}{*}{ Included } & \multirow[t]{3}{*}{ Included } \\
\hline & & & $\begin{array}{l}\text { Metformin } \\
750 \mathrm{mg} / \text { day }\end{array}$ & 106 & & & \\
\hline & & & $\begin{array}{l}\text { Metformin } \\
1500 \mathrm{mg} / \text { day }\end{array}$ & 106 & & & \\
\hline
\end{tabular}

$N / A$ not available; $N M A$ network meta-analysis

${ }^{a}$ Durations that were described in months were transformed into weeks

${ }^{\mathrm{b}}$ Number of patients available for primary outcome assessment

$150 \mathrm{mg} /$ day), and all six SGLT2i drugs (ipragliflozin 50 or $100 \mathrm{mg} /$ day, dapagliflozin 5 or $10 \mathrm{mg} /$ day, luseogliflozin 2.5 or $5 \mathrm{mg} /$ day, tofogliflozin $\quad 20 \mathrm{mg} /$ day, canagliflozin $100 \mathrm{mg} /$ day, and empagliflozin $10 \mathrm{mg} /$ day or $25 \mathrm{mg} /$ day) (Fig. 2A). A network graph including the selected studies and treatments for each outcome is shown in Fig. 2. For the reduction rate of elevated $\mathrm{HbA} 1 \mathrm{c}$ and the incidence rate of hypoglycemia, an RCT comparing glimepride $2 \mathrm{mg} /$ day and voglibose $0.9 \mathrm{mg} /$ day [55] was excluded because these treatments were not included in the network (Fig. 2). Network graphs for the sensitivity analyses of 31 studies after excluding those with a high risk of bias and 32 studies after excluding those in PPS or similar-to-PPS are shown in Supplementary Material Fig. S2.

\section{Change in the Hemoglobin A1c Level}

The mean difference in $\triangle \mathrm{HbA} 1 \mathrm{c}$ in response to metformin 750 or $1500 \mathrm{mg} /$ day (reference) for each treatment is shown as (mean $\Delta$ HbA1c (\%) for each intervention) - (mean $\Delta \mathrm{HbA} 1 \mathrm{c}(\%)$ for reference) in Fig. 3. When comparing metformin $1500 \mathrm{mg} /$ day with the 36 other treatments (except placebo) in the RCTs included in the meta-analysis, for all treatments except glimepiride $2 \mathrm{mg} /$ day and pioglitazone $45 \mathrm{mg} /$ day, the difference was a positive value, demonstrating that the reduction in HbA1c from baseline was smaller in patients treated with the treatment than in those treated with metformin $1500 \mathrm{mg} /$ day. The probability of observing inferior results compared with metformin $1500 \mathrm{mg} /$ day was $>0.975$ for 20 treatments, including 10 of 12 DPP-4i treatments selected for analysis. The difference from metformin $1500 \mathrm{mg} /$ day ranged from $0.40 \%$ for metformin $750 \mathrm{mg} /$ day to $0.96 \%$ for dapagliflozin $5 \mathrm{mg} /$ day among the 20 treatments and from $0.43 \%$ for sitagliptin $100 \mathrm{mg} /$ day to $0.60 \%$ for linagliptin $5 \mathrm{mg} /$ day among the $10 \mathrm{DPP}-4 \mathrm{i}$ treatments. For treatments with a negative value, the probability of showing superior effect compared with metformin $1500 \mathrm{mg}$ /day was 0.911 (the difference from metformin $1500 \mathrm{mg} /$ day: $-0.38 \%)$ for glimepiride $2 \mathrm{mg} /$ day and $0.540(-0.03 \%)$ for pioglitazone $45 \mathrm{mg}$ /day. Compared to metformin $750 \mathrm{mg} /$ day, four treatments showed superior outcomes with a probability of $>0.975$, and three treatments were inferior, showing a probability of $>0.975$.

In the analysis of the reduction rate of elevated HbA1c using claims data, the SD was calculated to be 1.21; this value was applied in further analysis. All 36 treatments showed a lower reduction rate than metformin $1500 \mathrm{mg} /$ day, and the probability of being inferior was $>0.975$ for 11 treatments (Fig. 3). 

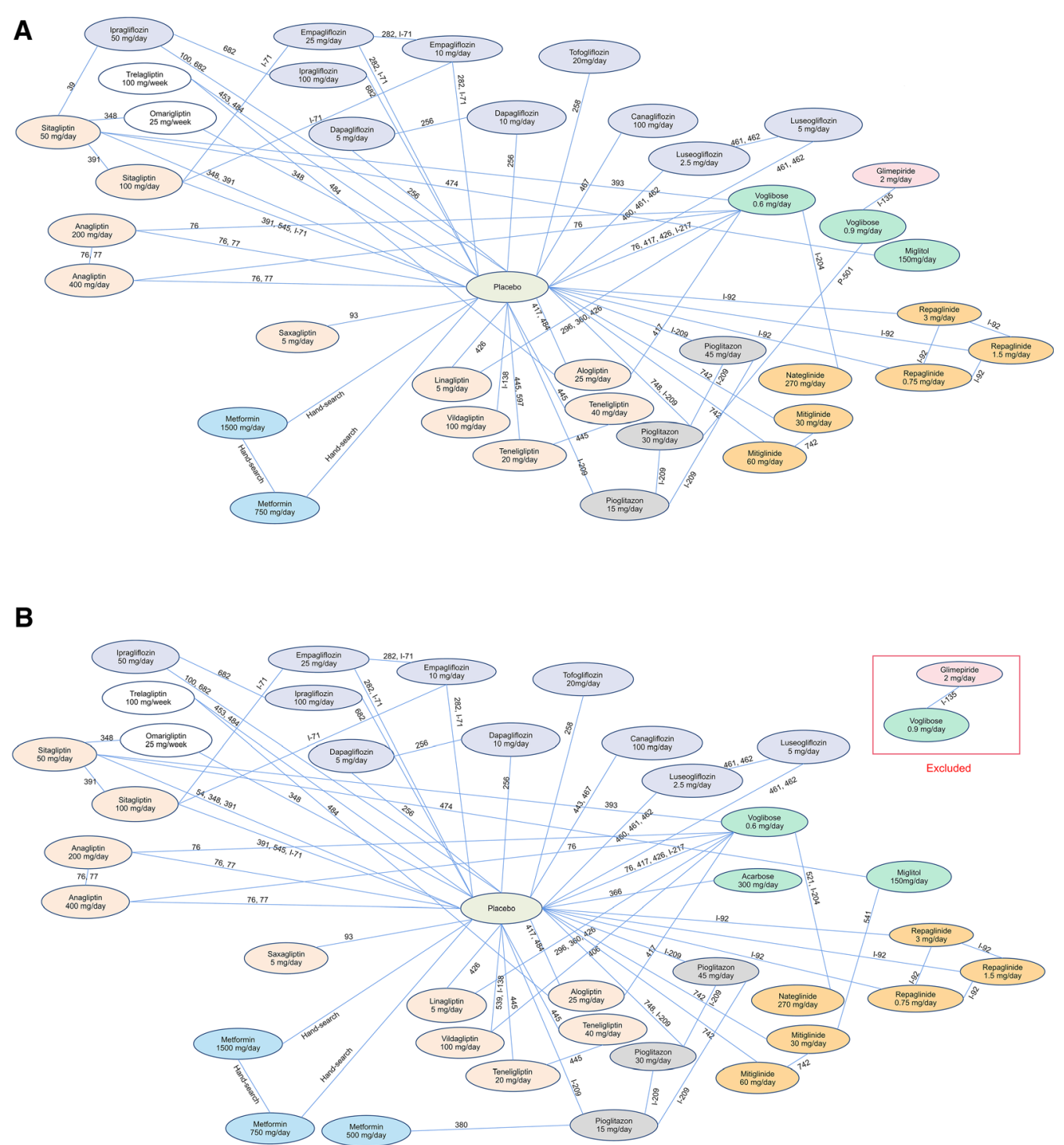

Fig. 2 Network graph of the treatments in the included randomized controlled trials for mean $(\Delta)$ hemoglobin Alc (a), reduction rate of elevated hemoglobin A1c (b),

Compared to metformin $750 \mathrm{mg} /$ day, only metformin $1500 \mathrm{mg} /$ day showed a higher reduction rate and no treatment showed a lower reduction rate, with a probability of $>0.975$.

\section{Incidence of Hypoglycemia}

The incidence rate of hypoglycemia was evaluated as a relative risk (RR) relative to a placebo; the incidence rate for each treatment was divided by the incidence rate for the placebo. The and incidence rate of hypoglycemia (c). The labels represent the study ID including each treatment arm

difference in RR compared with metformin 750 or $1500 \mathrm{mg} /$ day is shown in Fig. 3. Of the 31 treatments, only pioglitazone $30 \mathrm{mg} /$ day showed a lower RR $(<-0.01 \%$ as difference from metformin $1500 \mathrm{mg} /$ day), with a probability of $>0.975$, whereas most of other treatments showed a higher RR, including 23 and 21 treatments with a probability of $>0.975$, than metformin 1500 and $750 \mathrm{mg} /$ day, respectively. Glinide-based treatments, particularly, repaglinide, showed a higher difference in RR 
Fig. 2 continued

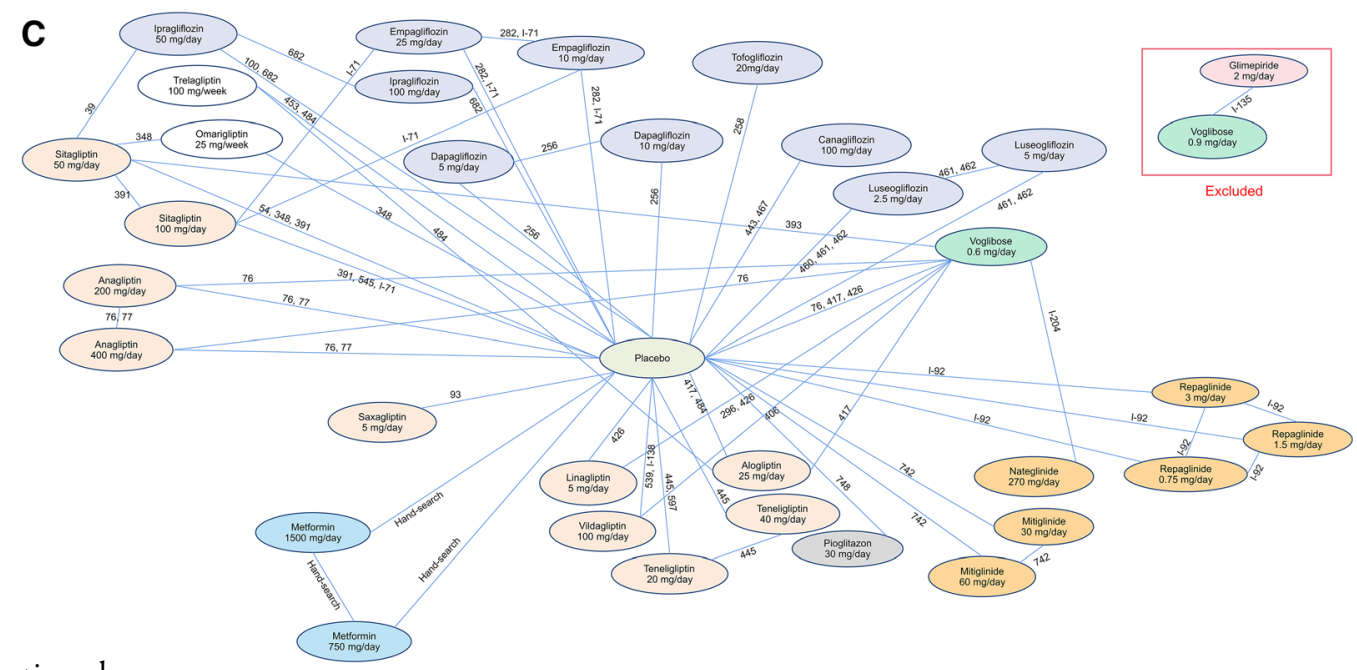

with increasing dosage. The difference in RR from metformin $1500 \mathrm{mg} /$ day was 49.39, 50.71, and 66.71 at $0.75,1.5$, and $3 \mathrm{mg} /$ day, respectively.

Supplementary Material Figure S3 shows the results of the $\Delta \mathrm{HbA} 1 \mathrm{c}$ sensitivity analyses. No significant differences were observed in the random-effect model analysis, analysis excluding high-bias-risk RCTs, and analysis excluding RCTs in PPS.

\section{DISCUSSION}

We performed a systematic review and an NMA to compare the efficacy of glycemic control and safety against the incidence of hypoglycemia of metformin at the maximum maintenance dose $(1500 \mathrm{mg} /$ day $)$ with those of other OADs approved in Japan in Japanese patients with T2D. In total, 46 RCTs were selected in the systematic review, and 37 studies, comprising 38 different types of treatments, including placebos, were selected for the NMA of $\Delta \mathrm{HbA} 1 \mathrm{c}$. Compared with metformin $1500 \mathrm{mg} /$ day, 20 treatments including metformin $750 \mathrm{mg}$ /day were significantly less effective in reducing the HbA1c level from baseline levels during a period of $\geq 12$ weeks. Two treatments (glimepiride $2 \mathrm{mg} /$ day and pioglitazone $45 \mathrm{mg}$ /day) showed greater mean reductions in HbA1c from baseline than metformin $1500 \mathrm{mg} /$ day, although these differences were not significant. In addition, we evaluated the glycemic control effect, as determined by the reduction rate of elevated HbA1c, to prevent a bias induced by the differences in baseline HbA1c level among studies. No treatment caused a higher mean reduction rate than metformin $1500 \mathrm{mg} /$ day, with a probability of $>0.975$ for 11 of the 36 treatments evaluated.

The incidence rate of hypoglycemia was reported by 36 studies. Particularly, a higher RR of hypoglycemia was observed with three repaglinide treatments, which were reported in one RCT [59]. The treatment duration in this RCT, which may be associated with the incidence of hypoglycemia, was 12 weeks, which was not longer than the other RCTs using different OADs. A possible reason for the higher hypoglycemia risk may be related to the structure of repaglinide, which is similar to that of sulfonylurea [60], which is known to induce hypoglycemia [61-63]. Hypoglycemia caused by sulfonylurea was not evaluated because of the lack of studies on this treatment. Notably, the RCT involving these three treatments [59] reported a relatively higher hypoglycemia incidence, even in the placebo group (5.6\%), than the other RCTs.

To assess the robustness of the main analysis, we conducted three sensitivity analyses on $\Delta$ HbA1c, applying a different model (randomeffect model) and excluding studies with a high risk of bias and those conducted in PPS. The 


\begin{tabular}{|c|c|c|c|c|c|c|c|c|c|c|c|c|c|}
\hline \multirow{2}{*}{\multicolumn{2}{|c|}{$\begin{array}{c}\text { Outcome } \\
\text { Intervention }\end{array}$}} & \multicolumn{4}{|c|}{ (1) $\triangle \mathrm{HbA} 1 \mathrm{c}$} & \multicolumn{4}{|c|}{$\begin{array}{l}\text { (2) Reduction rate } \\
\text { of elevated } \mathrm{HbA} 1 \mathrm{c}\end{array}$} & \multicolumn{4}{|c|}{$\begin{array}{l}\text { (3) Prevalence of } \\
\text { hypoglycemia }\end{array}$} \\
\hline & & \multicolumn{2}{|c|}{$\begin{array}{c}\text { To MET } \\
1500 \mathrm{mg} / \text { day }\end{array}$} & \multicolumn{2}{|c|}{$\begin{array}{c}\text { To MET } \\
750 \mathrm{mg} / \text { day }\end{array}$} & \multicolumn{2}{|c|}{$\begin{array}{c}\text { To MET } \\
1500 \mathrm{mg} / \text { day }\end{array}$} & \multicolumn{2}{|c|}{$\begin{array}{c}\text { To MET } \\
750 \mathrm{mg} / \text { day }\end{array}$} & \multicolumn{2}{|c|}{$\begin{array}{c}\text { To MET } \\
1500 \mathrm{mg} / \text { day }\end{array}$} & \multicolumn{2}{|c|}{$\begin{array}{c}\text { To MET } \\
750 \mathrm{mg} / \text { day }\end{array}$} \\
\hline Class & Type and dose & $\Delta$ & $\mathrm{P}$ & $\Delta$ & $\mathrm{P}$ & $\Delta$ & $\mathrm{P}$ & $\Delta$ & $\mathrm{P}$ & $\Delta$ & $\mathrm{P}$ & $\Delta$ & $\mathrm{P}$ \\
\hline$B G$ & Metformin $500 \mathrm{mg} /$ day & N/A & $\mathrm{N} / \mathrm{A}$ & N/A & N/A & -79.53 & 0.038 & -46.02 & 0.151 & N/A & N/A & N/A & N/A \\
\hline BG & Metformin & 0.40 & 0.000 & - & - & -33.51 & 0.017 & - & - & 0.03 & .512 & - & - \\
\hline BG & Metformin $1500 \mathrm{mg} /$ day & - & - & -0.40 & 1.000 & - & - & 33.51 & 0.983 & - & - & -0.03 & 0.488 \\
\hline TZD & Pioglitazone $15 \mathrm{mg} /$ day & 0.46 & 0.036 & 0.06 & 0.409 & -73.65 & 0.003 & -40.14 & 0.067 & $\mathrm{~N} / \mathrm{A}$ & $\mathrm{N} / \mathrm{A}$ & $\mathrm{N} / \mathrm{A}$ & $\mathrm{N} / \mathrm{A}$ \\
\hline TZD & Pioglitazone & 0.08 & 0.354 & -0.32 & 0.938 & -63.65 & 0.006 & -30.14 & 0.112 & -0.00 & 1.000 & -0.04 & 1.000 \\
\hline TZD & Pioglitazone $45 \mathrm{mg} /$ day & -0.03 & 0.540 & -0.43 & 0.931 & -59.10 & 0.021 & -25.59 & 0.189 & $\mathrm{~N} / \mathrm{A}$ & $\mathrm{N} / \mathrm{A}$ & $\mathrm{N} / \mathrm{A}$ & $\mathrm{N} / \mathrm{A}$ \\
\hline SU & gg/day & -0.38 & 0.911 & -0.78 & 1.000 & $\mathrm{~N} / \mathrm{A}$ & $\mathrm{N} / \mathrm{A}$ & $\mathrm{N} / \mathrm{A}$ & $\mathrm{N} / \mathrm{A}$ & $\mathrm{N} / \mathrm{A}$ & $\mathrm{N} / \mathrm{A}$ & $\mathrm{N} / \mathrm{A}$ & $\mathrm{N} / \mathrm{A}$ \\
\hline Glinide & g/day & 0.48 & 0.065 & 0.08 & 0.389 & -57.65 & 0.031 & -24.14 & .209 & 7.07 & 0.000 & 7.03 & .005 \\
\hline Glinide & & 0.44 & 0.075 & 0.04 & 0.440 & -52.99 & 0.051 & -19.48 & 0.273 & 18.96 & 0.000 & 3.92 & .002 \\
\hline Glinide & & 0.55 & & & & -39.86 & 0.081 & & & & 000 & & 003 \\
\hline Glinide & Repag & 0.39 & 0.030 & -0.01 & 0.514 & -24.82 & 0.225 & 8.69 & 594 & 49.39 & 0.000 & 49.35 & 0.000 \\
\hline Glinide & Repag & 0.13 & 0.251 & -0.27 & 0.924 & -6.94 & 0.410 & 26.57 & 0.776 & 50.71 & 0.000 & 50.68 & 0.000 \\
\hline Glinide & /day & 0.41 & 0.013 & 0.01 & 0.489 & -24.05 & 0.252 & 9.46 & 0.610 & 66.71 & 0.000 & 66.67 & 0.000 \\
\hline DPP-4i & & 0.47 & 0.007 & 0.07 & 0.317 & -38.81 & 0.064 & -5.30 & 0.408 & 1.79 & 0.001 & 1.75 & 0.029 \\
\hline DPP-4i & day & 0.54 & 0.001 & 0.14 & 0.207 & -41.00 & 0.059 & -7.49 & 0.360 & 2.18 & 0.006 & 15 & 0.015 \\
\hline DPP-4i & day & 0.45 & 0.006 & 0.05 & 0.361 & -40.29 & 0.049 & -6.78 & 0.384 & 5.31 & 0.001 & .28 & 0.008 \\
\hline DPP-4i & /day & 0.60 & 0.001 & 0.20 & 0.094 & -38.86 & 0.048 & -5.35 & 0.405 & 1.02 & 0.002 & .99 & 0.034 \\
\hline & eek & 0.47 & & 7 & & -36.00 & 0.074 & & & 4 & & & \\
\hline DPP-4i & day & 0.49 & 0.007 & 0.09 & 0.282 & -59.16 & 0.012 & -25.65 & 73 & .11 & 0.533 & .08 & 0.618 \\
\hline DPP-4i & Sitagliptin & 0.46 & 0.004 & 0.06 & 0.370 & -34.68 & 0.057 & -1.17 & 0.480 & 0.04 & 0.000 & 0.01 & 0.000 \\
\hline DPP-4i & Sitt & 0.43 & & 0.03 & & -25.83 & 0.129 & & 0.629 & .56 & 0.000 & 53 & 0.004 \\
\hline DPP-4i & Teneli & 0.50 & 0.002 & 0.10 & 0.275 & -40.21 & 0.073 & -6.70 & 0.398 & 3.95 & 0.000 & 3.92 & 0.008 \\
\hline DPP-4i & Tenelic & 0.36 & 0.033 & -0.04 & 0.586 & -26.18 & 0.160 & 7.33 & 0.601 & 10.82 & 0.000 & 10.79 & 0.000 \\
\hline DPP-4i & Trelagliptin 100 & 0.59 & 0.001 & 0.19 & 0.137 & -50.20 & 0.015 & -16.69 & 0.263 & 0.00 & 0.838 & -0.04 & 0.876 \\
\hline DPP-4i & Vildagliptin $100 \mathrm{mg} /$ day & 0.32 & 0.041 & -0.08 & 0.684 & -2.23 & 0.446 & 31.28 & 0.923 & 2.02 & 0.002 & 1.99 & 0.014 \\
\hline$\alpha-G I$ & Acarbose 3 & N/A & N/A & N/A & N/A & -83.47 & 0.056 & & & N/A & & $\mathrm{N} / \mathrm{A}$ & $\mathrm{N} / \mathrm{A}$ \\
\hline$\alpha-G I$ & $\mathrm{mg} /$ day & 0.85 & 0.004 & 0.45 & 0.100 & -39.14 & 0.164 & -5.63 & 0.456 & N/A & $\mathrm{N} / \mathrm{A}$ & N/A & $\mathrm{N} / \mathrm{A}$ \\
\hline$\alpha-G I$ & Voglibo & 0.90 & 0.000 & 0.50 & 0.002 & -69.38 & 0.001 & -35.87 & 0.047 & 3.34 & 0.000 & 3.31 & 0.005 \\
\hline$\alpha-G I$ & Voglibc & 0.19 & 0.240 & -0.21 & 0.795 & N/A & N/A & $\mathrm{N} / \mathrm{A}$ & $\mathrm{N} / \mathrm{A}$ & $\mathrm{N} / \mathrm{A}$ & $\mathrm{N} / \mathrm{A}$ & $\mathrm{N} / \mathrm{A}$ & $\mathrm{N} / \mathrm{A}$ \\
\hline SGLT-2i & anagliflozin 100 mg/day & 0.28 & 0.066 & -0.12 & 0.747 & -37.06 & 0.059 & -3.55 & 0.435 & 16.99 & 0.000 & 16.96 & 0.000 \\
\hline & & 0.96 & & & & & & & & & & 0.00 & 0.663 \\
\hline SGLT-2i & Dapagliflozin 10 mg/day & 0.92 & 0.000 & 0.52 & 0.002 & -60.29 & 0.014 & -26.78 & 0.168 & 6.63 & 0.000 & 6.59 & 0.003 \\
\hline SGLT-2i & Empagliflozin $10 \mathrm{mg} /$ day & 0.59 & 0.000 & 0.19 & 0.123 & -46.67 & 0.034 & -13.16 & 0.307 & 0.00 & 0.870 & -0.03 & 0.833 \\
\hline SGLT-2i & Empagliflozin 25 mg/day & 0.32 & 0.037 & -0.08 & 0.674 & -28.34 & 0.117 & 5.17 & 0.587 & 4.32 & 0.002 & 4.29 & 0.007 \\
\hline SGLT-2i & Ipragliflozin 50 mg/day & 0.06 & 0.366 & -0.34 & 0.976 & -36.02 & 0.080 & -2.51 & 0.463 & 1.77 & 0.003 & 1.73 & 0.022 \\
\hline SGLT-2i & Ipragliflozin 100 mg/day & 0.01 & 0.454 & -0.39 & 0.979 & -35.89 & 0.094 & -2.38 & 0.467 & 5.01 & 0.001 & 4.98 & 0.014 \\
\hline SGLT-2i & Luseogliflozin $2.5 \mathrm{mg} /$ day & 0.63 & 0.000 & 0.23 & 0.074 & -57.88 & 0.011 & -24.37 & 0.146 & 1.70 & 0.006 & 1.67 & 0.020 \\
\hline SGLT-2i & Luseogliflozin 5 mg/day & 0.57 & 0.001 & 0.17 & 0.149 & -52.26 & 0.021 & -18.75 & 0.233 & 3.00 & 0.002 & 2.97 & 0.021 \\
\hline SGLT-2i & Tofogliflozin $20 \mathrm{mg} /$ day & 0.32 & 0.052 & -0.08 & 0.658 & -48.58 & 0.062 & -15.07 & 0.304 & 0.00 & 0.882 & -0.04 & 0.874 \\
\hline
\end{tabular}

Fig. 3 Mean outcome difference of each treatment to metformin 1500 or $750 \mathrm{mg} /$ day (reference). $\Delta$ represents (mean $\Delta$ HbAlc (\%) for each intervention) - (mean $\triangle \mathrm{HbAlc}(\%)$ for reference) in (1), (reduction rate of elevated $\mathrm{HbAlc}(\%)$ for each intervention) - (reduction rate of elevated HbAlc (\%) for reference) in (2), and (relative risk of hypoglycemia to placebo for each intervention) - (relative risk of hypoglycemia to placebo for reference) in (3). P represents the probability ( 0 to 1 ) that each intervention is superior to reference. The value of

superiority/inferiority of the treatments compared to metformin 1500 or $750 \mathrm{mg}$ /day did not change, supporting the robustness of the main findings.

Regarding the dosages of metformin, although the $1500 \mathrm{mg}$ /day prescription showed significantly greater efficacy than many
P was rounded off to the third decimal place. Green and red colors indicate superiority and inferiority of the intervention $(p>0.975$ and $p<0.025)$ to the reference, respectively. $\alpha$-GI $\alpha$-glucosidase inhibitor, $B G$ biguanide, $D P P-4 i$ dipeptidyl peptidase-4 inhibitor, HbAlc hemoglobin A1c, MET metformin, SGLT-2i selective sodiumglucose transporter-2 inhibitor, $S U$ sulfonylurea, TZD thiazolidinedione

treatments, the efficacy of $750 \mathrm{mg} /$ day was not significantly higher than that of most of the other treatments. The risk of hypoglycemia did not widely differ between the dosages evaluated. Considering the difference in efficacy between these dosages and the current Japanese clinical situation in which metformin has been 
used at lower levels $(<1000 \mathrm{mg} /$ day on average), the use of metformin at $1500 \mathrm{mg} /$ day may be beneficial for patients with insufficient glycemic control.

There are only a few studies comparing the efficacy and safety between metformin and other OADs as a first-line treatment for patients with T2D using NMA, while many studies have compared the efficacy and safety among OADs as an add-on therapy to metformin, or for patients inadequately controlled with metformin. This is probably because metformin monotherapy is recommended as an initial treatment for patients in Western countries [1]. A 2016 NMA reported that, compared with metformin, sulfonylurea, thiazolidinediones, DPP- $4 \mathrm{i}$, and $\alpha$-glucosidase inhibitor were associated with a higher HbA1c level, and SGLT2i and meglitinides showed no significant difference in the HbA1c level at the end of treatment as a first-line monotherapy for $\geq 24$ weeks [64]. This study also reported that sulfonylurea is associated with a higher risk of hypoglycemia, and thiazolidinediones and DPP-4i were associated with a lower risk of hypoglycemia than metformin. This study compared treatments as drug class without being divided by drug type and dosage, and metformin dosage varied among RCTs, including those with higher dosages, such as 2500 or $3000 \mathrm{mg}$ /day as the maximum dose, compared with the approved dosage in Japan. Although we cannot compare the results with our results directly, a higher or similar effect of metformin in reducing HbA1c than that of other OADs is comparable between studies. A 2019 NMA compared 11 OADs by drug type (dapagliflozin, gliclazide, glimepiride, glipizide, glyburide, metformin, nateglinide, repaglinide, saxagliptin, sitagliptin, and vildagliptin), as a first-line monotherapy for patients with T2D, although various dosages were included for each treatment [65]. Repaglinide showed the highest efficacy in HbA1c reduction followed by gliclazide and metformin among the 11 OADs, and no significant difference was observed between repaglinide or gliclazide and metformin. The efficacy was significantly higher for metformin than for dapagliflozin, nateglinide, saxagliptin, sitagliptin, and vildagliptin. Although dosages in each drug were different, the results are consistent with our results for most of the drugs. Another NMA, comparing HbA1c reduction efficacy between metformin and sulfonylurea, SGLT2i, DPP-4i, or pioglitazone as a monotherapy reported that pioglitazone showed significantly lower efficacy after 12 weeks of treatment, whereas it showed higher efficacy after 104 weeks [66]. Our study did not show a significant difference in efficacy between pioglitazone with three different dosages and metformin; however, lower efficacy was suggested for pioglitazone as reduction rate of elevated HbA1c. Thus, pioglitazone might be associated with lower HbA1c-reducing effect at 12 weeks if baseline HbA1c level is adjusted. This study also reported that sulfonylurea showed a tendency of greater effect than metformin, although the difference was not significant, after 12 weeks of treatment. In our study, glimepiride $2 \mathrm{mg} /$ day, a sulfonylurea, showed a similar tendency to that in this previous study. Taken together, although there are several differences in the included RCTs, including types and dosages of drugs, treatment periods, and countries, as well as analysis methods, our results are in line with those of the previous NMA studies.

There were some limitations to this study. We targeted all OADs using approved dosages in Japan. However, not all available drugs were included, and the dosage was not the highest for some drugs because of a lack of RCTs meeting the eligibility criteria. The RCTs included in this study used diverse study designs, such as the targeted patients and treatment duration. Different classes of OADs were assessed, and each class of OAD has a different mechanism of action, which may have led to differences in the study designs. Differences in calendar year in which the RCTs were conducted may also impact the study design and results. Moreover, we included reports written in Japanese and English, whereas reports published in other languages were not included.

The studies were selected based on a comprehensive search of four databases and manual search of three clinical trial registries; however, the latter did not include RCTs conducted prior to a certain year (2000 for ClinicalTrials.gov and 2005 for JAPIC and UMIN). Hence, access to 
unpublished studies was restricted to the availability in (and limitations of) the registries. Differences in statistical analysis methods and data quality between the RCTs may have also affected our results. To reduce the risk inflicted by such differences, we assessed the risk of bias and effect of studies with a high risk of bias.

Finally, we evaluated the outcomes at 12 weeks or the closest period $>12$ weeks from the initiation of interventions. It is because 12 weeks is considered to be the time when the change in HbA1c level stabilizes and optimal period to evaluate the outcomes, and majority of RCTs evaluated the outcomes at the point. Selecting this period allowed us to compare a variety of treatments; however, the results may not reflect the efficacy and safety of long-term treatment. In addition, as we included only RCTs, the treatment situation might be different in RCTs from that in real-world settings. Consequently, further research is needed to compare the outcomes among treatments in real-world settings.

\section{CONCLUSIONS}

A systematic review and an NMA were performed to compare the efficacy and safety of metformin at $1500 \mathrm{mg} /$ day with those of the other glucose-lowering agents available to Japanese patients with T2D. The results suggest that metformin $1500 \mathrm{mg} /$ day has mostly a preferable efficacy and safety profile compared with the other OADs approved in Japan. The information provided in this study could shed insights for physicians to choose a suitable firstline treatment for their patients with T2D among the variety of options available in Japan. Further research is needed to compare the efficacy and safety in real-world settings.

\section{ACKNOWLEDGEMENTS}

The authors wish to thank Kenji Baba (Sumitomo Dainippon Pharma Co., Ltd.) for his contribution to the study design.
Funding. Sponsorship for this study and Rapid Service Fee and Open Access fee was funded by Sumitomo Dainippon Pharma Co., Ltd.

Editorial Assistance. Editorial assistance in the preparation of this article was provided by Editage (http://www.editage.com), which was funded by Sumitomo Dainippon Pharma Co., Ltd.

Authorship. All named authors meet the International Committee of Medical Journal Editors (ICMJE) criteria for authorship for this article, take responsibility for the integrity of the work as a whole, and have approved this version of the manuscript to be published.

Authors' Contributions. RN and MT contributed to the study conception. All authors contributed to the study design and interpretation of data. KI contributed to systematic review and network meta-analysis. TT contributed to systematic review and develop the first draft of the manuscript. All authors commented on previous versions of the manuscript. All authors read and approved the final manuscript.

Prior Presentation. Parts of this work were presented at the 64th Annual Meeting of the Japan Diabetes Society (virtual meeting), May 20-22, 2021.

Disclosures. Rimei Nishimura has received honoraria from Astellas Pharma Inc., Nippon Boehringer Ingelheim Co., Ltd., Eli Lilly Japan K.K., Kissei Pharmaceutical Co., Ltd., Medtronic Japan Co., Ltd., MSD, Novartis Pharma K.K., Novo Nordisk Pharma Ltd., Sanofi K.K., Sumitomo Dainippon Pharma Co., Ltd., and Takeda Pharmaceutical Co., Ltd. He has also received a grant from the Japan Diabetes Foundation and Nippon Boehringer Ingelheim Co., Ltd. Mayumi Taniguchi is an employee of Sumitomo Dainippon Pharma Co., Ltd. Tomomi Takeshima and Kosuke Iwasaki are employees of Milliman Inc., which has received consultancy fees from Sumitomo Dainippon Pharma Co., Ltd. 
Compliance with Ethics Guidelines. This study was based on previously conducted studies and does not contain any new studies with human participants or animals performed by any of the authors. Therefore, ethical approval and informed consent were not required. The protocol of this study was registered at PROSPERO (CRD42020160779).

Data Availability. The data used in this study are published data from clinical trials, which are included in this article and supplementary material.

Open Access. This article is licensed under a Creative Commons Attribution-NonCommercial 4.0 International License, which permits any non-commercial use, sharing, adaptation, distribution and reproduction in any medium or format, as long as you give appropriate credit to the original author(s) and the source, provide a link to the Creative Commons licence, and indicate if changes were made. The images or other third party material in this article are included in the article's Creative Commons licence, unless indicated otherwise in a credit line to the material. If material is not included in the article's Creative Commons licence and your intended use is not permitted by statutory regulation or exceeds the permitted use, you will need to obtain permission directly from the copyright holder. To view a copy of this licence, visit http:// creativecommons.org/licenses/by-nc/4.0/.

\section{REFERENCES}

1. Davies MJ, D'Alessio DA, Fradkin J, et al. Management of hyperglycemia in type 2 diabetes, 2018. A consensus report by the American Diabetes Association (ADA) and the European Association for the Study of Diabetes (EASD). Diabetes Care. 2018;2018(41):2669-701.

2. Araki E, Goto A, Kondo T, et al. Japanese clinical practice guideline for diabetes 2019. Diabetol Int. 2020;11:165-223.

3. Odawara M, Kawamori R, Tajima N, et al. Longterm treatment study of global standard dose metformin in Japanese patients with type 2 diabetes mellitus. Diabetol Int. 2017;8:286-95.

4. Kameda T, Kumamaru H, Nishimura S, Kohsaka S, Miyata $\mathrm{H}$. Use of oral antidiabetic drugs in Japanese working-age patients with type 2 diabetes mellitus: dosing pattern for metformin initiators. Curr Med Res Opin. 2020;36:749-56.

5. Tamura Y, Kato H, Hiroi S, et al. A study on the prescription status of metformin and DPP-4 inhibitors in patients with type 2 diabetes mellitus in Japan. An analysis of data from health insurance administrative claims. Ther Res. 2017;38:1231-7 (Japanese).

6. Metgluco $250 \mathrm{mg}$. Approval application form. Sumitomo Dainippon Pharma Co., Ltd; 2008. https://www.pmda.go.jp/drugs/2010/P201000009/ index.html. Accessed 20 August 2021. (Japanese)

7. Higgins JPT, Thomas J, Chandler J, et al. (editors) Cochrane Handbook for Systematic Reviews of Interventions version 6.1 (updated September 2020). Cochrane; 2020. https://training.cochrane. org/handbook/archive/v6.1. Accessed 20 Aug 2021.

8. Hutton B, Salanti G, Caldwell DM, et al. The PRISMA extension statement for reporting of systematic reviews incorporating network meta-analyses of health care interventions: checklist and explanations. Ann Intern Med. 2015;162:777-84.

9. Edited by Julian PT Higgins JS, Matthew J Page, Jonathan AC Sterne on behalf of the RoB2 Development Group. Revised Cochrane risk-of-bias tool for randomized trials (RoB 2): RoB2 Development Group; 2019. https://methods.cochrane.org/riskbias-2. Accessed 20 Aug 2021.

10. Kashiwagi A. Development of the HbA1c international standardization of HbA1c measurement in Japan Diabetes Society. Rinsho Byori. 2013;61: 585-93 (Japanese).

11. Committee of the Japan Diabetes Society on the Diagnostic Criteria of Diabetes Mellitus, Seino Y, Nanjo K, Tajima N, et al. Report of the committee on the classification and diagnostic criteria of diabetes mellitus. J Diabetes Investig. 2010;1:212-28.

12. International Expert Committee. International Expert Committee report on the role of the A1C assay in the diagnosis of diabetes. Diabetes Care. 2009;32:1327-34.

13. DeFronzo RA, Stonehouse AH, Han J, Wintle ME. Relationship of baseline HbA1c and efficacy of current glucose-lowering therapies: a meta-analysis of randomized clinical trials. Diabet Med. 2010;27: 309-17. 
14. Monotherapy study of MP-513 in patients with type 2 diabetes. ClinicalTrials.gov identifier: NCT00998881. Updated February 21, 2014. https:// clinicaltrials.gov/ct2/show/NCT00998881. Accessed 20 Aug 2021.

15. A phase II confirmatory study of SMP-862 monotherapy in patients with type 2 diabetes mellitus. Japan Pharmaceutical Information Center identifier: JapicCTI-050090. Updated July 21, 2010. https://www.clinicaltrials.jp/cti-user/trial/ ShowDirect.jsp?japicId=JapicCTI-050090. Accessed 20 Aug 2021.

16. Kato T, Sawai Y, Kanayama H, et al. Comparative study of low-dose pioglitazone or metformin treatment in Japanese diabetic patients with metabolic syndrome. Exp Clin Endocrinol Diabetes. 2009;117: 593-9.

17. Fujitaka K, Otani H, Jo F, et al. Comparison of metabolic profile and adiponectin level with pioglitazone versus voglibose in patients with type2 diabetes mellitus associated with metabolic syndrome. Endocr J. 2011;58:425-32.

18. Mikada A, Narita T, Yokoyama H, et al. Effects of miglitol, sitagliptin, and initial combination therapy with both on plasma incretin responses to a mixed meal and visceral fat in over-weight Japanese patients with type 2 diabetes. "The MASTER randomized, controlled trial." Diabetes Res Clin Pract. 2014;106:538-47.

19. Tsurutani Y, Nakai K, Inoue K, et al. Comparative study of the effects of ipragliflozin and sitagliptin on multiple metabolic variables in Japanese patients with type 2 diabetes: a multicentre, randomized, prospective, open-label, active-controlled study. Diabetes Obes Metab. 2018;20:2675-9.

20. Kaku K. Dose-ranging study of anagliptin in Japanese patients with type 2 diabetes: a multi-centre, randomized, placebo-controlled, double-blind, parallel-group study. Jpn Pharmacol Ther. 2012;40: 973-84 (Japanese).

21. Hotta N, Kakuta H, Sano T, et al. Long-term effect of acarbose on glycaemic control in non-insulin-dependent diabetes mellitus: a placebo-controlled double-blind study. Diabetes Med. 1993;10:134-8.

22. Iwamoto Y, Tajima N, Kadowaki T, et al. Efficacy and safety of sitagliptin monotherapy compared with voglibose in Japanese patients with type 2 diabetes: a randomized, double-blind trial. Diabetes Obes Metab. 2010;12:613-22.

23. Yokoyama H, Inoue $T$, Node K. Effect of insulinunstimulated diabetic therapy with miglitol on serum cystatin C level and its clinical significance. Diabetes Res Clin Pract. 2009;83:77-82.
24. Tanaka SI, Origasa H, Akanuma Y, Kikuchi M. Evaluation of glucose lowering effects of mitiglinide in type 2 diabetes patients: placebo-controlled, parallel group comparative dose-response study. Jpn Pharmacol Ther. 2007;35:S5-21 (Japanese).

25. Kosaka K, Kikuchi M, Tarui S, et al. Phase-III study of a novel hypoglycemic agent, AY4166, on NIDDM patients in Japan. (1): a-glucosidase inhibitor, voglibose-controlled multicenter double blind study. Clin Pharmacol Therapy. 1997;7:699-727 (Japanese).

26. Kaneko T, Baba S, Toyoda T, et al. Dose setting test of $\mathrm{AD}-4833$ for non-insulin dependent diabetes mellitus (NIDDM) by only diet therapy. Double blind intergroup comparative studies by 4 doses. Jpn J Clin Exp Med. 1997;74:1250-77.

27. Yamada $Y$, Terauchi $Y$, Watada $H$, et al. Efficacy and safety of GPR119 agonist DS-8500a in Japanese patients with type 2 diabetes: a randomized, double-blind, placebo-controlled, 12-week study. Adv Ther. 2018;35:367-81.

28. Kaku K. Efficacy and safety of anagliptin in Japanese patients with type 2 diabetes: a multi-centre, randomized, placebo- and active comparator-controlled, double-blind, parallel-group study. Jpn Pharmacol Ther. 2012;40:985-95 (Japanese).

29. Seino Y. Efficacy and safety of saxagliptin in Japanese patients with type 2 diabetes: two multi-centre, randomized, double-blind, placebo-controlled studies. Jpn Pharmacol Ther. 2014;42:503-18 (Japanese).

30. Kashiwagi A, Kazuta K, Takinami Y, Yoshida S, Utsuno A, Nagase I. Ipragliflozin improves glycemic control in Japanese patients with type 2 diabetes mellitus: the BRIGHTEN study: BRIGHTEN: doubleblind randomized study of ipragliflozin to show its efficacy as monotherapy in T2DM patients. Diabetol Int. 2015;6:8-18.

31. Kaku K, Kiyosue A, Inoue S, et al. Efficacy and safety of dapagliflozin monotherapy in Japanese patients with type 2 diabetes inadequately controlled by diet and exercise. Diabetes Obes Metab. 2014;16: 1102-10.

32. Kaku K, Watada H, Iwamoto Y, et al. Efficacy and safety of monotherapy with the novel sodium/glucose cotransporter-2 inhibitor tofogliflozin in Japanese patients with type 2 diabetes mellitus: a combined Phase 2 and 3 randomized, placebocontrolled, double-blind, parallel-group comparative study. Cardiovasc Diabetol. 2014;13:65.

33. Kadowaki T, Haneda M, Inagaki N, et al. Empagliflozin monotherapy in Japanese patients with type 2 diabetes mellitus: a randomized, 12-week, double- 
blind, placebo-controlled, phase II trial. Adv Ther. 2014;31:621-38.

34. Fujitani Y, Fujimoto S, Takahashi K, et al. Effects of linagliptin monotherapy compared with voglibose on postprandial blood glucose responses in Japanese patients with type 2 diabetes: linagliptin study of effects on postprandial blood glucose (L-STEP). Diabetes Res Clin Pract. 2016;121:146-56.

35. Gantz I, Okamoto T, Ito Y, et al. A randomized, placebo- and sitagliptin-controlled trial of the safety and efficacy of omarigliptin, a once-weekly dipeptidyl peptidase-4 inhibitor, in Japanese patients with type 2 diabetes. Diabetes Obes Metab. 2017;19:1602-9.

36. Satoh H, Ohira T, Moriya C, et al. Effects of linagliptin vs. voglibose on daily glucose excursions during continuous glucose monitoring of Japanese type 2 diabetes patients (L-CGM): a randomized, open-label, two-arm, parallel comparative trial. Diabetes Metab. 2017;43:550-3.

37. Kato $T$, Inoue $T$, Node K. Postprandial endothelial dysfunction in subjects with new-onset type 2 diabetes: an acarbose and nateglinide comparative study. Cardiovasc Diabetol. 2010;9:12.

38. Iwamoto $\mathrm{Y}$, Taniguchi $\mathrm{T}$, Nonaka $\mathrm{K}$, et al. Doseranging efficacy of sitagliptin, a dipeptidyl peptidase- 4 inhibitor, in Japanese patients with type 2 diabetes mellitus. Endocr J. 2010;57:383-94.

39. Iwamoto Y, Kashiwagi A, Yamada N, et al. Efficacy and safety of vildagliptin and voglibose in Japanese patients with type 2 diabetes: a 12 -week, randomized, double-blind, active-controlled study. Diabetes Obes Metab. 2010;12:700-8.

40. Seino Y, Fujita T, Hiroi S, Hirayama M, Kaku K. Efficacy and safety of alogliptin in Japanese patients with type 2 diabetes mellitus: a randomized, double-blind, dose-ranging comparison with placebo, followed by a long-term extension study. Curr Med Res Opin. 2011;27:1781-92.

41. Kawamori R, Inagaki N, Araki E, et al. Linagliptin monotherapy provides superior glycaemic control versus placebo or voglibose with comparable safety in Japanese patients with type 2 diabetes: a randomized, placebo and active comparator-controlled, double-blind study. Diabetes Obes Metab. 2012;14:348-57.

42. Inagaki N, Kondo K, Yoshinari $\mathrm{T}$, Maruyama N, Susuta Y, Kuki H. Efficacy and safety of canagliflozin in Japanese patients with type 2 diabetes: a randomized, double-blind, placebo-controlled, 12-week study. Diabetes Obes Metab. 2013;15: 1136-45.
43. Kadowaki T, Kondo K. Efficacy, safety and dose-response relationship of teneligliptin, a dipeptidyl peptidase- 4 inhibitor, in Japanese patients with type 2 diabetes mellitus. Diabetes Obes Metab. 2013;15:810-8.

44. Inagaki N, Onouchi H, Sano H, Funao N, Kuroda S, Kaku K. SYR-472, a novel once-weekly dipeptidyl peptidase-4 (DPP-4) inhibitor, in type 2 diabetes mellitus: a phase 2, randomised, double-blind, placebo-controlled trial. Lancet Diabetes Endocrinol. $2014 ; 2: 125-32$.

45. Seino Y, Sasaki T, Fukatsu A, Ubukata M, Sakai S, Samukawa Y. Efficacy and safety of luseogliflozin as monotherapy in Japanese patients with type 2 diabetes mellitus: a randomized, double-blind, placebo-controlled, phase 3 study. Curr Med Res Opin. 2014;30:1245-55.

46. Seino Y, Sasaki T, Fukatsu A, Ubukata M, Sakai S, Samukawa Y. Dose-finding study of luseogliflozin in Japanese patients with type 2 diabetes mellitus: a 12-week, randomized, double-blind, placebo-controlled, phase II study. Curr Med Res Opin. 2014;30: 1231-44.

47. Seino Y, Sasaki T, Fukatsu A, Sakai S, Samukawa Y. Efficacy and safety of luseogliflozin monotherapy in Japanese patients with type 2 diabetes mellitus: a 12-week, randomized, placebo-controlled, phase II study. Curr Med Res Opin. 2014;30:1219-30.

48. Inagaki N, Kondo $\mathrm{K}$, Yoshinari $\mathrm{T}$, Takahashi $\mathrm{N}$, Susuta Y, Kuki H. Efficacy and safety of canagliflozin monotherapy in Japanese patients with type 2 diabetes inadequately controlled with diet and exercise: a 24-week, randomized, double-blind, placebo-controlled, phase III study. Expert Opin Pharmacother. 2014;15:1501-15.

49. Inagaki $\mathrm{N}$, Onouchi $\mathrm{H}$, Maezawa $\mathrm{H}$, Kuroda S, Kaku K. Once-weekly trelagliptin versus daily alogliptin in Japanese patients with type 2 diabetes: a randomised, double-blind, phase 3, non-inferiority study. Lancet Diabetes Endocrinol. 2015;3:191-7.

50. Kurebayashi S, Watada H, Tanaka Y, Kawasumi M, Kawamori R, Hirose T. Efficacy and adverse effects of nateglinide in early type 2 diabetes. Comparison with voglibose in a cross-over study. Endocr J. 2006;53:213-7.

51. Kikuchi M, Abe N, Kato M, Terao S, Mimori N, Tachibana H. Vildagliptin dose-dependently improves glycemic control in Japanese patients with type 2 diabetes mellitus. Diabetes Res Clin Pract. 2009;83:233-40.

52. Nonaka K, Kakikawa T, Sato A, et al. Efficacy and safety of sitagliptin monotherapy in Japanese 
patients with type 2 diabetes. Diabetes Res Clin Pract. 2008;79:291-8.

53. Kashiwagi A, Kazuta K, Yoshida S, Nagase I. Randomized, placebo-controlled, double-blind glycemic control trial of novel sodium-dependent glucose cotransporter 2 inhibitor ipragliflozin in Japanese patients with type 2 diabetes mellitus. J Diabetes Investig. 2014;5:382-91.

54. Kaneko T. Clinical evaluation of an insulin resistance-improving agent, $\mathrm{AD}-4833$, in patients with non-insulin-dependent diabetes mellitus receiving diet therapy alone: a double-blind placebo-controlled study. Jpn J Clin Exp Med. 1997;74: 1491-514 (Japanese).

55. Koh N, Hayashi K, Hosokawa T. Effects of glimepiride on blood glucose control and oxidative stress in patients with type 2 diabetes-a study by nagoya medical association. J New Rem Clin. 2010;59: 363-73 (Japanese).

56. Kikuchi $\mathrm{M}$, Iwamoto $\mathrm{Y}$, Inagaki $\mathrm{N}$, Yoshioka $\mathrm{T}$, Mimori N, Ebina H. Clinical evaluation of vildagliptin in patients with type 2 diabetes: a multicenter, randomized, double-blind, placebo-controlled, parallel group comparative study. J New Rem Clin. 2010;59:121-36 (Japanese).

57. Kamiya F, Hayashi Y, Takeuchi T. The clinical usefulness of chronic administration of AO-128 for non-insulin dependent diabetes mellitus. Comparative test of double blind experiment for which a placebo is used. J Adult Dis. 1992;22:573-91.

58. Koiwai K, Inoue S, Shiki K, Taniguchi A. Efficacy and safety of empagliflozin monotherapy in Japanese patients with type 2 diabetes: a sub-analysis of randomized, 24-week, double-blind global clinical trial. Prog Med. 2015;35:1231-42 (Japanese).

59. Kawamori R, Kaku K, Hanafusa T, Oikawa T, Kageyama S, Hotta N. Dose response study of rapidacting insulin secretagogue repaglinide
(SUREPOST ${ }^{\circledR}$ ) monotherapy in patients with type 2 diabetes mellitus late phase II randomized doubleblind study. J Clin Therap Med. 2014;30:113-27 (Japanese).

60. Hansen AM, Christensen IT, Hansen JB, Carr RD, Ashcroft FM, Wahl P. Differential interactions of nateglinide and repaglinide on the human beta-cell sulphonylurea receptor 1. Diabetes. 2002;51: 2789-95.

61. The Japan Diabetes Society. [Treatment Guide for Diabetes 2018-2019]. Tokyo: Bunkodo; 2018. (Japanese).

62. UK Prospective Diabetes Study (UKPDS) Group. Effect of intensive blood-glucose control with metformin on complications in overweight patients with type 2 diabetes (UKPDS 34). Lancet. 1998;352: 854-65.

63. UK Prospective Diabetes Study (UKPDS) Group. Intensive blood-glucose control with sulphonylureas or insulin compared with conventional treatment and risk of complications in patients with type 2 diabetes (UKPDS 33). Lancet. 1998;352: 837-53.

64. Palmer SC, Mavridis D, Nicolucci A, et al. Comparison of clinical outcomes and adverse events associated with glucose-lowering drugs in patients with type 2 diabetes: a meta-analysis. JAMA. 2016;316:313-24.

65. Jia Y, Lao Y, Zhu H, Li N, Leung SW. Is metformin still the most efficacious first-line oral hypoglycaemic drug in treating type 2 diabetes? A network meta-analysis of randomized controlled trials. Obes Rev. 2019;20:1-12.

66. Mannucci E, Naletto L, Vaccaro G, et al. Efficacy and safety of glucose-lowering agents in patients with type 2 diabetes: a network meta-analysis of randomized, active comparator-controlled trials. Nutr Metab Cardiovasc Dis. 2021;31:1027-34. 